\author{
D. A. Samaras, E. Eleftheriadou, K. Theodoropoulos \& G. Karetsos
}

\title{
Contribution to the flora of Mt Goulinas (Sterea Ellas, Greece)
}

\begin{abstract}
Samaras, D. A., Eleftheriadou, E., Theodoropoulos K. \& Karetsos, G.: Contribution to the flora of Mt Goulinas (Sterea Ellas, Greece). — Fl. Medit. 27: 241-266. 2017. — ISSN: 1120-4052 printed, 2240-4538 online.

Mt Goulinas is located in the NW part of Sterea Ellas in south-central Greece. A first floristic inventory of the mountain is presented based on field work and literature records. The known vascular flora of the study area consists of 487 taxa out of which 305 taxa are reported for the first time. Among these Euphorbia phymatosperma subsp. cernua and Elymus caninus are new to Sterea Ellas.

Key words: vascular plants, floristic inventory, south-central Greece.
\end{abstract}

\section{Introduction}

Over the last decades an intensive floristic research carried out in the mountains of Sterea Ellas in south-central Greece (Dimopoulos \& al. 2013). Despite that big effort, the botanical exploration has been focused almost entirely on the high mountains, leaving outside extensive coastal, lowland and lower mountain areas. Mt Goulinas is one of these low mountains, with an elevation of less than $1500 \mathrm{~m}$ a.s.l., that has been "floristically ignored". Although Mt Goulinas presents a phytogeographical interest due to its geographical position and habitat richness, the only published floristic study of the area is that of Willing \& Willing $(2006,2008)$. Two more studies of the vegetation and phytogeography of the mountains of Sterea Ellas, including Mt Goulinas, have been published by Barbéro \& Quézel (1976) and Phitos (1960). A few records of Orchids have been published by Künkele (1983), Hölzinger \& al. (1985) and Tsiftsis \& Antonopoulos (2017).

This study tries to fill the gap in knowledge of the incompletely explored flora of Mt Goulinas by presenting a floristic catalogue of all vascular plant records of the first author together with literature records. 


\section{Materials and methods}

Study area - Mt Goulinas is located in south-central Greece (Sterea Ellas), in the west part of Fthiotida prefecture. The mountain lies between $38^{\circ} 49^{\prime} 20^{\prime \prime}$ and $38^{\circ} 56^{\prime} 32^{\prime \prime} \mathrm{N}$ of latitude and $21^{\circ} 57^{\prime} 26^{\prime \prime}$ and $22^{\circ} 12^{\prime} 05^{\prime \prime}$ E of longitude, and covers an area of 16,210 ha that ranges from 100 to $1459 \mathrm{~m}$ in elevation (Fig. 1). The study area is restricted among the rivers Sperchios, Inachos and Roustianitis. The dominant geological formation is flysch (consisted mainly of sandstone), while limestones and scree slope deposits occur, to a small extent, in the central part of the mountain, close to the highest summit (Kallergis \& al. 1970). The climate is Mediterranean with wet, mild winters and dry, hot summers. According to Köppen climatic classification, the lower elevations belong to the Csa type, while the higher to the Csb (Samaras \& al. 2008).

The most abundant vegetation type in the study area is the deciduous oak forest and scrub, dominated by Quercus pubescens in the lower elevations and Q. frainetto in the middle and higher elevations. Other deciduous trees like $Q$. petraea subsp. polycarpa, Castanea sativa and Ostrya carpinifolia can be found locally on north facing slopes in clumps or in mixture with $Q$. frainetto. In the higher elevations, above the oak forests, on north and north-east facing slopes can be found the Greek fir (Abies cephalonica) forest. In the highest elevations, above the timberline, forest fires, overgrazing and illegal logging

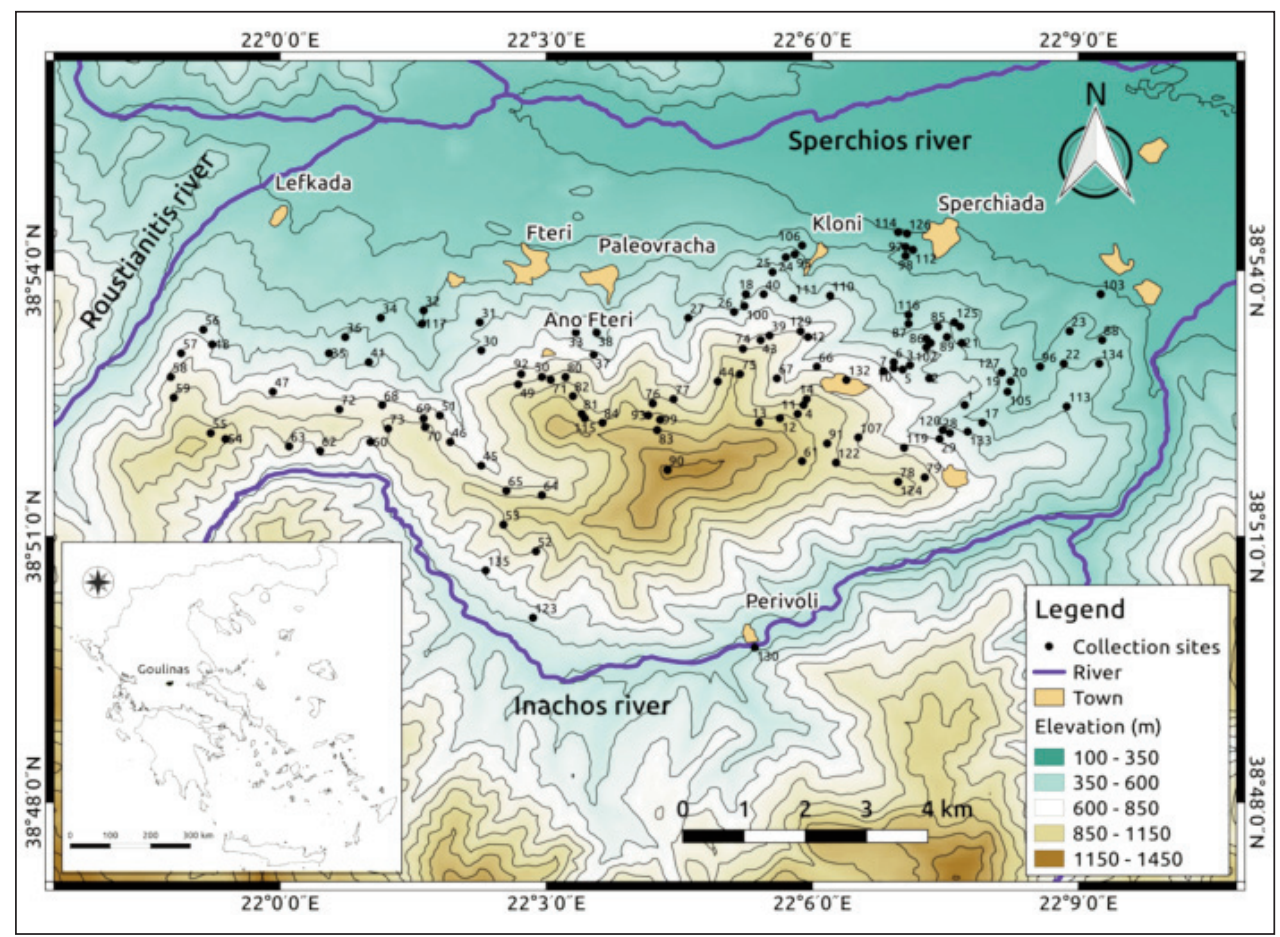

Fig. 1. Map of the study area and collection localities. 
have lead to degradation and the replacement of forests by prickly juniper shrublands and anthropogenic, montane, dry grasslands. Extensive evergreen scrub vegetation (pseudomacchie) dominated by $Q$. coccifera occur in the lowlands and foothills of Mt Goulinas mainly in the eastern part of the study area. Along streams and rivers the riparian vegetation consists mainly of the tree species Platanus orientalis, Salix alba, Populus alba and Alnus glutinosa and the shrubs Nerium oleander and Vitex agnus-castus.

Data collection and analysis - The floristic catalogue is based on collections and field observations carried out by the first author during spring, summer and autumn of 2002-2003 and 2005-2007. Published records are also included in the catalogue. Plant specimens that were collected in the field are stored in the herbarium of the Laboratory of Forest Botany-Geobotany, in the Aristotle University of Thessaloniki, Greece (TAUF). Plant identification was based on the main national and European Floras (Tutin \& al. 1968-1980, 1993; Strid 1986; Strid \& Tan 1991, 1997, 2002) and selected taxonomic literature (Zohary \& Heller 1984; Krendl 1986-1987; Boyce 1988, 1994; Zielinski 1990; Christensen 1992; Baumann \& al. 2006). The nomenclature mainly follows Dimopoulos \& al. (2013). Accepted names are set in boldface italics in the floristic catalogue. Families, genera, species and subspecies are arranged alphabetically within the four main taxonomic groups: Pteridophyta, Gymnospermae, Dicotyledoneae and Monocotyledoneae. Life-form categories of taxa are coded according to Raunkiaer's classification system as was modified by Dimopoulos \& al. (2013). Chorological types of taxa are based on the system established for the checklist of vascular plants of Greece (Dimopoulos \& al. 2013).

Collection localities - Collection sites (Fig. 1) are coded with numbers (locality codes). The locality codes of all specimens collected in the field are given in the floristic catalogue, in boldface, before the collection number. Information about the date of collection, the exact coordinates, the altitude and the habitat of the site is given below:

1: $38^{\circ} 52^{\prime} 29^{\prime \prime} / 22^{\circ} 07^{\prime} 43^{\prime \prime}-570 \mathrm{~m}$ a.s.1. - 5/1/2002 - Forest of Quercus pubescens

2: $38^{\circ} 52^{\prime} 47^{\prime \prime} / 22^{\circ} 07^{\prime} 19^{\prime \prime}-610 \mathrm{~m}$ a.s.1. $-5 / 4 / 2002$ - Forest of $Q$. frainetto

3: $38^{\circ} 52^{\prime} 56^{\prime \prime} / 22^{\circ} 07^{\prime} 06^{\prime \prime}-640 \mathrm{~m}$ a.s.1. - 5/7-27/2002, 7/10/2002 - Mixed forest of Q. frainettoQ. pubescens

4: $38^{\circ} 52^{\prime} 29^{\prime \prime} / 22^{\circ} 05^{\prime} 54^{\prime \prime}$ - $990 \mathrm{~m}$ a.s.1. - 7/9/2002 - Forest of Abies cephalonica

5: $38^{\circ} 52^{\prime} 53^{\prime \prime} / 22^{\circ} 07^{\prime} 01^{\prime \prime}-650 \mathrm{~m}$ a.s.1. - 7/11/2002 - Forest of $Q$. frainetto

6: $38^{\circ} 52^{\prime} 58^{\prime \prime} / 22^{\circ} 06^{\prime} 55^{\prime \prime}-630 \mathrm{~m}$ a.s.l. - 7/12/2002 - Forest of $Q$. frainetto

7: $38^{\circ} 52^{\prime}$ '52" / 22 $02^{\circ}$ ' 48" - 660-680 m a.s.1. - 7/13-16/2002, 6/22/2003 - Forest of $Q$. frainetto

10: $38^{\circ} 52^{\prime} 54^{\prime \prime} / 22^{\circ} 06^{\prime} 55^{\prime \prime}-660-670 \mathrm{~m}$ a.s.1. - 7/18-19/2002 - Mixed forest of $Q$. frainetto- $Q$. pubescens

11: $38^{\circ} 52^{\prime} 23^{\prime \prime} / 22^{\circ} 05^{\prime} 50^{\prime \prime}$ - $1010 \mathrm{~m}$ a.s.1. - 7/22-26/2002 - Forest of Abies cephalonica

12: $38^{\circ} 52^{\prime} 20^{\prime \prime} / 22^{\circ} 05^{\prime} 38^{\prime \prime}-1010 \mathrm{~m}$ a.s.1. - 6/19/2003 - Forest of $A$. cephalonica

13: $38^{\circ} 52^{\prime} 17^{\prime \prime} / 22^{\circ} 05^{\prime} 24^{\prime \prime}-1020$ m a.s.l. - 6/21/2003 - Forest of $A$. cephalonica

14: $38^{\circ} 52^{\prime} 33^{\prime \prime} / 22^{\circ} 05^{\prime} 56^{\prime \prime}$ - 960-990 m a.s.1. - 6/23-24/2003 - Forest of $A$. cephalonica

15: $38^{\circ} 53^{\prime} 22^{\prime \prime} / 22^{\circ} 07^{\prime} 40^{\prime \prime}-460 \mathrm{~m}$ a.s.1. - 4/29/2005 - Forest of $Q$. pubescens

16: $38^{\circ} 53^{\prime} 11^{\prime \prime} / 22^{\circ} 07$ ' $20^{\prime \prime}$ - $560 \mathrm{~m}$ a.s.1. - 4/30/2005, 04/19/2006 - Meadow next to mixed forest of $Q$. pubescens- $Q$. frainetto / Mixed forest of $Q$. pubescens- $Q$. frainetto

17: $38^{\circ} 52^{\prime} 17^{\prime \prime} / 22^{\circ} 07^{\prime} 55^{\prime \prime}-520 \mathrm{~m}$ a.s.l. - 5/2/2005 - Forest of $Q$. frainetto 
18: $38^{\circ} 53^{\prime} 44^{\prime \prime} / 22^{\circ} 05^{\prime} 15^{\prime \prime}-570 \mathrm{~m}$ a.s.1. - 5/3/2005 - Forest of $Q$. frainetto/Roadside in $Q$. frainetto forest

19: $38^{\circ} 52^{\prime} 51^{\prime \prime} / 22^{\circ} 08^{\prime} 08^{\prime \prime}-460 \mathrm{~m}$ a.s.1. - 5/4/2005 - Forest of $Q$. frainetto

20: $38^{\circ} 52^{\prime} 45^{\prime \prime} / 22^{\circ} 08^{\prime} 14^{\prime \prime}-450 \mathrm{~m}$ a.s.1. - 5/4/2005 - Mixed forest of Q. pubescens-Q. frainetto

21: $38^{\circ} 53^{\prime} 11^{\prime \prime} / 22^{\circ} 07^{\prime} 41^{\prime \prime}-460-470 \mathrm{~m}$ a.s.1. - 5/5/2005 - Forest of $Q$. pubescens

22: $38^{\circ} 52^{\prime} 57^{\prime \prime} / 22^{\circ} 08^{\prime} 50^{\prime \prime}-380 \mathrm{~m}$ a.s.1. - 6/7/2005 - Forest of $Q$. pubescens

23: $38^{\circ} 53^{\prime} 19^{\prime \prime} / 22^{\circ} 08^{\prime} 54^{\prime \prime}-320 \mathrm{~m}$ a.s.1. - 6/7/2005 - Forest of $Q$. pubescens / Clearing in $Q$. pubescens forest

24: $38^{\circ} 54^{\prime} 09^{\prime \prime} / 22^{\circ} 05^{\prime} 42^{\prime \prime}-470 \mathrm{~m}$ a.s.1. - 6/8/2005 - Forest of $Q$. frainetto

25: $38^{\circ} 53^{\prime} 59^{\prime \prime} / 22^{\circ} 05^{\prime} 33^{\prime \prime}-530 \mathrm{~m}$ a.s.1. - 6/8/2005 - Forest of $Q$. frainetto

26: $38^{\circ} 53^{\prime} 32^{\prime \prime} / 22^{\circ} 05^{\prime} 07^{\prime \prime}-630 \mathrm{~m}$ a.s.1. - 6/9/2005 - Forest of $Q$. frainetto

27: $38^{\circ} 53^{\prime} 28^{\prime \prime} / 22^{\circ} 04^{\prime} 36^{\prime \prime}$ - 580 m a.s.1. - 6/9/2005 - Forest of Q. frainetto

28: $38^{\circ} 52^{\prime} 10^{\prime \prime} / 22^{\circ} 07^{\prime} 33^{\prime \prime}$ - $650 \mathrm{~m}$ a.s.1. - 6/10/2005, 4/28/2006 - Forest of $Q$. frainetto

29: $38^{\circ} 52^{\prime} 06^{\prime \prime} / 22^{\circ} 07^{\prime} 26^{\prime \prime}-690 \mathrm{~m}$ a.s.l. - 6/10/2005 - Forest of $Q$. frainetto

30: $38^{\circ} 53^{\prime} 06^{\prime \prime} / 22^{\circ} 02^{\prime} 16^{\prime \prime}-640 \mathrm{~m}$ a.s.1. - 6/11/2005 - Forest of $Q$. frainetto

31: $38^{\circ} 53^{\prime} 25^{\prime \prime} / 22^{\circ} 02^{\prime} 15^{\prime \prime}-560 \mathrm{~m}$ a.s.1. - 6/11/2005 - Forest of $Q$. frainetto

32: $38^{\circ} 53^{\prime} 33^{\prime \prime} / 22^{\circ} 01^{\prime} 37^{\prime \prime}-400 \mathrm{~m}$ a.s.1. - 6/14/2005 - Forest of $Q$. frainetto

33: $38^{\circ} 53^{\prime} 18^{\prime \prime} / 22^{\circ} 03^{\prime} 20^{\prime \prime}-680 \mathrm{~m}$ a.s.1. - 6/15/2005 - Forest of Q. frainetto

34: $38^{\circ} 53^{\prime} 28^{\prime \prime} / 22^{\circ} 01^{\prime} 08^{\prime \prime}$ - $440 \mathrm{~m}$ a.s.1. - 6/15/2005 - Forest of $Q$. frainetto

35: $38^{\circ} 53^{\prime} 04^{\prime \prime} / 22^{\circ} 00^{\prime} 33^{\prime \prime}-560$ m a.s.1. - 6/16/2005 - Forest of $Q$. petraea/Roadside in Quercus petraea forest

36: $38^{\circ} 53^{\prime} 15^{\prime \prime} / 22^{\circ} 00^{\prime} 44^{\prime \prime}-530 \mathrm{~m}$ a.s.1. - 6/16/2005 - Forest of $Q$. frainetto

37: $38^{\circ} 53^{\prime} 03^{\prime \prime} / 22^{\circ} 03^{\prime} 32^{\prime \prime}-710 \mathrm{~m}$ a.s.1. - 6/17/2005 - Mixed forest of $Q$. frainetto-Castanea sativa-Q. petraea

38: $38^{\circ} 53^{\prime} 18^{\prime \prime} / 22^{\circ} 03^{\prime} 34^{\prime \prime}-590 \mathrm{~m}$ a.s.1. - 6/17/2005 - Forest of $Q$. frainetto

39: $38^{\circ} 53^{\prime} 16^{\prime \prime} / 22^{\circ} 05^{\prime} 31^{\prime \prime}-710 \mathrm{~m}$ a.s.1. - 6/18/2005 - Mixed forest of Q. frainetto-Q. petraea

40: $38^{\circ} 53^{\prime} 44^{\prime \prime} / 22^{\circ} 05^{\prime} 27^{\prime \prime}-630 \mathrm{~m}$ a.s.1. - 6/18/2005 - Forest of $Q$. frainetto

41: $38^{\circ} 52^{\prime} 58^{\prime \prime} / 22^{\circ} 01^{\prime} 00^{\prime \prime}-570 \mathrm{~m}$ a.s.1. - 6/20/2005 - Forest of $Q$. frainetto

42: $38^{\circ} 53^{\prime} 15^{\prime \prime} / 22^{\circ} 05^{\prime} 57^{\prime \prime}-780 \mathrm{~m}$ a.s.1. - 6/21/2005 - Forest of $Q$. frainetto

43: $38^{\circ} 53^{\prime} 13^{\prime \prime} / 22^{\circ} 05^{\prime} 25^{\prime \prime}$ - $840 \mathrm{~m}$ a.s.1. - 6/21/2005 - Forest of $Q$. petraea

44: $38^{\circ} 52^{\prime} 45^{\prime \prime} / 22^{\circ} 04^{\prime} 56^{\prime \prime}-870 \mathrm{~m}$ a.s.l. - 6/22/2005 - Forest of $A$. cephalonica

45: $38^{\circ} 51^{\prime} 48^{\prime \prime} / 22^{\circ} 02^{\prime} 16^{\prime \prime}-800 \mathrm{~m}$ a.s.1. - 6/23/2005 - Forest of $Q$. frainetto

46: $38^{\circ} 52^{\prime} 04^{\prime \prime} / 22^{\circ} 01^{\prime} 55^{\prime \prime}-840 \mathrm{~m}$ a.s.1. - 6/23/2005 - Forest of Castanea sativa

47: $38^{\circ} 52^{\prime} 38^{\prime \prime} / 21^{\circ} 59^{\prime} 55^{\prime \prime}-770 \mathrm{~m}$ a.s.1. - 6/24/2005 - Forest of $Q$. frainetto

48: $38^{\circ} 53^{\prime} 10^{\prime \prime} / 21^{\circ} 59^{\prime} 14^{\prime \prime}-710 \mathrm{~m}$ a.s.1. - 6/24/2005 - Forest of $Q$. frainetto

49: $38^{\circ} 52^{\prime} 43^{\prime \prime} / 22^{\circ} 02^{\prime} 41^{\prime \prime}-850-920$ m a.s.1. - 6/25/2005 - Mixed forest of $Q$. petraea- $Q$. frainettoCastanea sativa

50: $38^{\circ} 52^{\prime} 48^{\prime \prime} / 22^{\circ} 02^{\prime} 57^{\prime \prime}$ - 900 m a.s.1. - 6/25/2005 - Mixed forest of Castanea sativa-Q. frainettoQ. petraea

51: $38^{\circ} 52^{\prime} 22^{\prime \prime} / 22^{\circ} 01^{\prime} 48^{\prime \prime}-790 \mathrm{~m}$ a.s.l. - 6/26/2005 - Mixed forest of Ostrya carpinifolia-Q. frainetto

52: $38^{\circ} 50^{\prime} 50^{\prime \prime} / 22^{\circ} 02^{\prime} 53^{\prime \prime}-900 \mathrm{~m}$ a.s.1. - 6/28/2005 - Forest of $Q$. frainetto

53: $38^{\circ} 51^{\prime} 08^{\prime \prime} / 22^{\circ} 02^{\prime} 31^{\prime \prime}-1000 \mathrm{~m}$ a.s.l. - 6/28/2005 - Forest of $Q$. frainetto

54: $38^{\circ} 52^{\prime} 06^{\prime \prime} / 21^{\circ} 59^{\prime} 23^{\prime \prime}-1030 \mathrm{~m}$ a.s.1. - 6/29/2005 - Forest of $Q$. frainetto

55: $38^{\circ} 52^{\prime} 10^{\prime \prime} / 21^{\circ} 59^{\prime} 13^{\prime \prime}-1070 \mathrm{~m}$ a.s.1. - 6/29/2005 - Mixed forest of Q. petraea-Abies cephalonica-Castanea sativa

56: $38^{\circ} 53^{\prime} 20^{\prime \prime} / 21^{\circ} 59^{\prime} 08^{\prime \prime}-660 \mathrm{~m}$ a.s.l. - 7/3/2005 - Forest of $Q$. frainetto

57: $38^{\circ} 53^{\prime} 04^{\prime \prime} / 21^{\circ} 58^{\prime} 53^{\prime \prime}-750 \mathrm{~m}$ a.s.1. - 7/4/2005 - Forest of $Q$. frainetto

58: $38^{\circ} 52^{\prime} 48^{\prime \prime} / 21^{\circ} 58^{\prime} 46^{\prime \prime}-840 \mathrm{~m}$ a.s.1. - 7/4/2005 - Forest of $Q$. frainetto 
59: $38^{\circ} 52^{\prime} 34^{\prime \prime} / 21^{\circ} 58^{\prime} 48^{\prime \prime}-910 \mathrm{~m}$ a.s.1. - 7/4/2005 - Mixed forest of Q. petraea-Castanea sativa-Q. frainetto

60: $38^{\circ} 52^{\prime} 04^{\prime \prime} / 22^{\circ} 01^{\prime} 01^{\prime \prime}-800 \mathrm{~m}$ a.s.1. - 7/5/2005 - Forest of $Q$. frainetto

61: $38^{\circ} 51^{\prime} 51^{\prime \prime} / 22^{\circ} 05^{\prime} 53^{\prime \prime}-750 \mathrm{~m}$ a.s.1. - 7/5/2005 - Forest of $Q$. frainetto

62: $38^{\circ} 51^{\prime} 58^{\prime \prime} / 22^{\circ} 00^{\prime} 27^{\prime \prime}-830 \mathrm{~m}$ a.s.1. - 7/6/2005 - Forest of $Q$. frainetto

63: $38^{\circ} 52^{\prime} 01^{\prime \prime} / 22^{\circ} 00^{\prime} 06^{\prime \prime}-860 \mathrm{~m}$ a.s.1. - 7/6/2005 - Forest of Q. frainetto

64: $38^{\circ} 51^{\prime} 28^{\prime \prime} / 22^{\circ} 02^{\prime} 57^{\prime \prime}$ - 970 m a.s.l. - 7/7/2005 - Mixed stand of Platanus orientalis- $Q$. frainetto / Roadside in $Q$. frainetto forest

65: $38^{\circ} 51^{\prime} 31^{\prime \prime} / 22^{\circ} 02^{\prime} 33^{\prime \prime}-920 \mathrm{~m}$ a.s.1. - 7/7/2005 - Mixed forest of $Q$. petraea-Q. frainetto

66: $38^{\circ} 52^{\prime} 55^{\prime \prime} / 22^{\circ} 06^{\prime} 03^{\prime \prime}-810 \mathrm{~m}$ a.s.1. - 7/8/2005 - Forest of Abies cephalonica

67: $38^{\circ} 52^{\prime} 47^{\prime \prime} / 22^{\circ} 05^{\prime} 36^{\prime \prime}-840 \mathrm{~m}$ a.s.1. - 7/8/2005 - Forest of $A$. cephalonica

68: $38^{\circ} 52^{\prime} 29^{\prime \prime} / 22^{\circ} 01^{\prime} 09^{\prime \prime}-810 \mathrm{~m}$ a.s.1. - 7/10/2005 - Forest of $Q$. frainetto

69: $38^{\circ} 52^{\prime} 20^{\prime \prime} / 22^{\circ} 01^{\prime} 37^{\prime \prime}-860 \mathrm{~m}$ a.s.1. - 7/10/2005 - Mixed forest of C. sativa-Q. petraea

70: $38^{\circ} 52^{\prime} 14 " / 22^{\circ} 01$ ' $38^{\prime \prime}$ - $910 \mathrm{~m}$ a.s.1. - 7/11/2005 - Mixed forest of $C$. sativa-Q. frainetto

71: $38^{\circ} 52^{\prime}$ 46" / 22 $02^{\circ}$ ' 03" - $920 \mathrm{~m}$ a.s.1. - 7/11/2005 - Mixed forest of C. sativa-Q. petraea

72: $38^{\circ} 52^{\prime} 26^{\prime \prime} / 22^{\circ} 00^{\prime} 40^{\prime \prime}-870 \mathrm{~m}$ a.s.1. - 7/12/2005 - Forest of $Q$. frainetto

73: $38^{\circ} 52^{\prime} 13^{\prime \prime} / 22^{\circ} 01 " 13 "-900 \mathrm{~m}$ a.s.1. - 7/12/2005 - Forest of $Q$. frainetto

74: $38^{\circ} 53^{\prime} 07^{\prime \prime} / 22^{\circ} 05^{\prime} 13^{\prime \prime}$ - $910 \mathrm{~m}$ a.s.1. - 7/13/2005 - Mixed forest of $A$. cephalonica- $Q$. petraea 75: $38^{\circ} 52^{\prime} 50^{\prime \prime} / 22^{\circ} 05^{\prime} 11^{\prime \prime}$ - $1070 \mathrm{~m}$ a.s.1. - 7/13/2005 - Mixed forest of $A$. cephalonica- $Q$. petraea

76: $38^{\circ} 52^{\prime} 30^{\prime \prime} / 22^{\circ} 04$ ' 12" - 1030-1040 m a.s.1. - 7/18/2005 - Roadside / Clearing in A. cephalonica$Q$. petraea forest / Mixed forest of A. cephalonica-Q. petraea

77: $38^{\circ} 52^{\prime} 33^{\prime \prime} / 22^{\circ} 04 " 26^{\prime \prime}-980 \mathrm{~m}$ a.s.1. - 7/18/2005 - Forest of $A$. cephalonica

78: $38^{\circ} 51^{\prime} 37^{\prime \prime} / 22^{\circ} 06^{\prime} 58^{\prime \prime}$ - 1060 m a.s.1. - 7/19/2005, 4/19/2006 - Forest of Q. frainetto / Clearing in $Q$. frainetto forest

79: $38^{\circ} 51^{\prime} 40^{\prime \prime} / 22^{\circ} 07$ ' 16" - $950 \mathrm{~m}$ a.s.1. - 7/19/2005, 4/19/2006 - Forest of Q. frainetto

80: $38^{\circ} 52^{\prime} 48^{\prime \prime} / 22^{\circ} 03$ ' 13 " - 980 m a.s.1. - 7/20/2005 - Forest of $Q$. petraea/Mixed forest of $Q$. petraea-Castanea sativa- $Q$. frainetto

81: $38^{\circ} 52^{\prime} 23^{\prime \prime} / 22^{\circ} 03^{\prime} 24^{\prime \prime}$ - 1100 m a.s.1. - 7/20-23/2005 - Forest of $Q$. petraea / Mixed forest of Q. petraea-Castanea sativa

82: $38^{\circ} 52^{\prime} 35^{\prime \prime} / 22^{\circ} 03^{\prime} 18^{\prime \prime}-1030 \mathrm{~m}$ a.s.1. - 7/21/2005 - Forest of $Q$. petraea

83: $38^{\circ} 52^{\prime} 12^{\prime \prime} / 22^{\circ} 04^{\prime} 15^{\prime \prime}-1200$ m a.s.l. - 7/22/2005 - Forest of $A$. cephalonica

84: $38^{\circ} 52^{\prime} 17^{\prime \prime} / 22^{\circ} 03^{\prime} 38^{\prime \prime}-1110 \mathrm{~m}$ a.s.1. - 7/22/2005 - Mixed forest of $Q$. petraea-A. cephalonica

85: $38^{\circ} 53^{\prime} 22^{\prime \prime} / 22^{\circ} 07^{\prime} 25^{\prime \prime}$ - $520 \mathrm{~m}$ a.s.1. - 4/26/2006 - Forest of $Q$. pubescens

86: $38^{\circ} 53^{\prime} 14^{\prime \prime} / 22^{\circ} 07^{\prime} 17^{\prime \prime}-540 \mathrm{~m}$ a.s.l. - 4/26/2006 - Forest of $Q$. pubescens

87: $38^{\circ} 53^{\prime} 24^{\prime \prime} / 22^{\circ} 07^{\prime} 05^{\prime \prime}$ - $470 \mathrm{~m}$ a.s.1. - 4/27/2006 - Forest of Q. pubescens

88: $38^{\circ} 53^{\prime} 13^{\prime \prime} / 22^{\circ} 09^{\prime} 16^{\prime \prime}-350 \mathrm{~m}$ a.s.1. - 4/27/2006 - Forest of $Q$. pubescens

89: $38^{\circ} 53^{\prime} 15^{\prime \prime} / 22^{\circ} 07^{\prime} 31^{\prime \prime}$ - $530 \mathrm{~m}$ a.s.1. - 4/29/2006 - Forest of $Q$. pubescens

90: $38^{\circ} 51^{\prime} 45^{\prime \prime} / 22^{\circ} 04^{\prime} 22^{\prime \prime}-1460$ m a.s.l. - 6/12/2007 - Rocky slope

91: $38^{\circ} 52^{\prime} 03^{\prime \prime} / 22^{\circ} 06^{\prime} 10^{\prime \prime}-1060$ m a.s.l. - 6/12/2007 - Rocky meadow

92: $38^{\circ} 52^{\prime} 50^{\prime \prime} / 22^{\circ} 02^{\prime} 43^{\prime \prime}-850 \mathrm{~m}$ a.s.l. - 6/25/2005 - Mixed forest of $Q$. frainetto- $Q$. petraeaCastanea sativa

93: $38^{\circ} 52^{\prime} 22^{\prime \prime} / 22^{\circ} 04^{\prime} 09^{\prime \prime}$ - 1100 m a.s.1. - 7/22/2005 - Roadside in A. cephalonica forest / Mixed forest of $A$. cephalonica- $Q$. petraea

95: $38^{\circ} 54^{\prime} 11^{\prime \prime} / 22^{\circ} 05^{\prime} 48^{\prime \prime}-430 \mathrm{~m}$ a.s.1. - 5/7/2005 - Roadside

96: $38^{\circ} 52^{\prime} 55^{\prime \prime} / 22^{\circ} 08^{\prime} 34^{\prime \prime}-310 \mathrm{~m}$ a.s.1. - 5/4/2005 - Roadside in meadow

97: $38^{\circ} 54^{\prime} 16^{\prime \prime} / 22^{\circ} 07^{\prime} 03^{\prime \prime}-280 \mathrm{~m}$ a.s.1. - 4/24/2006 - Roadside

98: $38^{\circ} 54^{\prime} 10^{\prime \prime} / 22^{\circ} 07^{\prime} 03^{\prime \prime}$ - $250 \mathrm{~m}$ a.s.l. - 7/5/2005 - Roadside in meadow

99: $38^{\circ} 52^{\prime} 19^{\prime \prime} / 22^{\circ} 04^{\prime} 17^{\prime \prime}$ - 1140 m a.s.1. - 7/22/2005 - Forest of $A$. cephalonica

100: $38^{\circ} 53^{\prime} 36^{\prime \prime} / 22^{\circ} 05^{\prime} 14^{\prime \prime}-610-620 \mathrm{~m}$ a.s.1. - 5/7/2005 - Roadside in Q. frainetto forest 
102: $38^{\circ} 53^{\prime} 08^{\prime \prime} / 22^{\circ} 07^{\prime} 17^{\prime \prime}-560$ m a.s.l. - 4/30/2005 - Ravine

103: $38^{\circ} 53^{\prime} 44^{\prime \prime} / 22^{\circ} 09^{\prime} 15^{\prime \prime}-260 \mathrm{~m}$ a.s.1. - 4/18/2006 - Abandoned field / Open shrubland with

Q. pubescens- $Q$. coccifera-Pyrus spinosa-Juniperus oxycedrus

104: 490-580 m a.s.1. - 4/25/2003 - Margins of oak forest / Clearing in oak forest / Roadside

105: $38^{\circ} 52^{\prime} 38^{\prime \prime} / 22^{\circ} 08^{\prime} 12^{\prime \prime}$ - $470 \mathrm{~m}$ a.s.l. - 5/4/2005 - Meadow / Margins of oak forest

106: $38^{\circ} 54^{\prime} 17^{\prime \prime} / 22^{\circ} 05^{\prime} 53^{\prime \prime}$ - 360 m a.s.l. - 5/7/2005 - Roadside

107: $38^{\circ} 52^{\prime} 07^{\prime \prime} / 22^{\circ} 06^{\prime} 31^{\prime \prime}$ - 910 m a.s.1. - 4/19/2006 - Roadside

110: $38^{\circ} 53^{\prime} 43^{\prime \prime} / 22^{\circ} 06^{\prime} 12^{\prime \prime}$ - 580 m a.s.1. - 5/7/2005 - Roadside

111: $38^{\circ} 53^{\prime} 41^{\prime \prime} / 22^{\circ} 05^{\prime} 47^{\prime \prime}-600$ m a.s.1. - 5/7/2005 - Roadside in Q. frainetto forest / Margins of $Q$. frainetto forest

112: $38^{\circ} 54^{\prime} 14^{\prime \prime} / 22^{\circ} 07^{\prime} 08^{\prime \prime}-230 \mathrm{~m}$ a.s.l. - 4/24/2006 - Meadow next to river

113: $38^{\circ} 52^{\prime} 28^{\prime \prime} / 22^{\circ} 08^{\prime} 52^{\prime \prime}-4 / 27 / 2006$ - Roadside

114: $38^{\circ} 54^{\prime} 26^{\prime \prime} / 22^{\circ} 06^{\prime} 58^{\prime \prime}-200 \mathrm{~m}$ a.s.l. - 4/24/2006 - Reforestation of Pinus-along path

115: $38^{\circ} 52^{\prime} 20^{\prime \prime} / 22^{\circ} 03^{\prime} 26^{\prime \prime}$ - $1110 \mathrm{~m}$ a.s.1. - 7/23/2005 - Forest of $Q$. petraea

116: $38^{\circ} 53^{\prime} 30^{\prime \prime} / 22^{\circ} 07^{\prime} 05^{\prime \prime}$ - $430 \mathrm{~m}$ a.s.l. - 4/25/2006 - Roadside / Abandoned forest road

117: $38^{\circ} 53^{\prime} 24^{\prime \prime} / 22^{\circ} 01^{\prime} 36^{\prime \prime}-380 \mathrm{~m}$ a.s.1. - 6/14/2005 - Roadside in a riverside

119: $38^{\circ} 52^{\prime} 00^{\prime \prime} / 22^{\circ} 07^{\prime} 02^{\prime \prime}-850 \mathrm{~m}$ a.s.l. $-5 / 2 / 2005$ - Roadside in a ravine

120: $38^{\circ} 52^{\prime} 12^{\prime \prime} / 22^{\circ} 07$ ' $28^{\prime \prime}$ - $640 \mathrm{~m}$ a.s.1. - 5/2/2005 - Meadow / Q. frainetto forest / Margins of

$Q$. frainetto forest - damp place, next to water tank

122: $38^{\circ} 51^{\prime} 50 "$ " $22^{\circ} 06^{\prime} 16^{\prime \prime}$ - 990 m a.s.l. - 4/19/2006 - Roadside next to stream

123: $38^{\circ} 50^{\prime} 05^{\prime \prime} / 22^{\circ} 02^{\prime} 51^{\prime \prime}$ - 640 m a.s.l - 5/6/2005 - Roadside

124: $38^{\circ} 51^{\prime} 37^{\prime \prime} / 22^{\circ} 06^{\prime} 58^{\prime \prime}$ - 1100 m a.s.l. - 4/19/2006 - Forest of $Q$. frainetto

125: $38^{\circ} 53^{\prime} 25^{\prime \prime} / 22^{\circ} 07^{\prime} 36^{\prime \prime}-480 \mathrm{~m}$ a.s.l. - 4/25/2006 - Meadow / Margins of Q. pubescens forest

/ Clearing in $Q$. pubescens forest

126: $38^{\circ} 54^{\prime} 25^{\prime \prime} / 22^{\circ} 07^{\prime} 04^{\prime \prime}-210$ m a.s.l. - 4/24/2006 - Reforestation of Pinus-along path

127: $38^{\circ} 52^{\prime} 51^{\prime \prime} / 22^{\circ} 08^{\prime} 08^{\prime \prime}-450 \mathrm{~m}$ a.s.l. - 5/4/2005 - Clearing in $Q$. pubescens forest

129: $38^{\circ} 53^{\prime} 19^{\prime \prime} / 22^{\circ} 05^{\prime} 52^{\prime \prime}-780 \mathrm{~m}$ a.s.l. - 6/21/2005 - Mixed forest of $Q$. frainetto- $Q$. petraea

130: $38^{\circ} 49^{\prime} 45^{\prime \prime} / 22^{\circ} 05^{\prime} 21^{\prime \prime}$ - $460 \mathrm{~m}$ a.s.l. - 5/6/2005 - Riverside

132: $38^{\circ} 52^{\prime} 46^{\prime \prime} / 22^{\circ} 06^{\prime} 23^{\prime \prime}-800 \mathrm{~m}$ a.s.l. - 7/19/2005 - Roadside

133: $38^{\circ} 52^{\prime} 11^{\prime \prime} / 22^{\circ} 07^{\prime} 45^{\prime \prime}-580 \mathrm{~m}$ a.s.l. $-5 / 2 / 2005$ - Abandoned forest road

134: $38^{\circ} 52^{\prime} 57^{\prime \prime} / 22^{\circ} 09^{\prime} 14$ " -410 m a.s.l. - 4/27/2006 - Stream

135: $38^{\circ} 50^{\prime} 37^{\prime \prime} / 22^{\circ} 02$ ' $19^{\prime \prime}-650$ m a.s.l. - 6/6/2005 - Roadside-Stream

\section{Results}

Floristic catalogue - The following abbreviations and symbols are used in the floristic catalogue:

+ : new record

* : literature report not confirmed

$\bullet$ : greek endemic

obs. : observed in the field (no collection available)

s.lat. : sensu lato

s.n. : without number

$\{=.$.$\} : synonym$

$\{“ . . "\}$ : misapplied name or misidentification

Sam. : leg. D. Samaras (TAUF), followed by collection number

B\&Q : Barbéro \& Quézel 
W\&W : Willing \& Willing

$\mathrm{H}:$ Hölzinger \& al.

$\mathrm{K}$ : Künkele

T\&A : Tsiftsis \& Antonopoulos

\section{PTERIDOPHYTA}

ASPLENIACEAE

+ Asplenium adiantum-nigrum L. - H, Pt. - 5 (Sam.1), 24 (Sam.610), 87 (Sam.1189) Asplenium onopteris L. - H, ME - B\&Q (1976:15), 87 (Sam.1188), 92 (Sam.847)

+ Asplenium trichomanes subsp. quadrivalens D. E. Mey. - H, EA - 92 (Sam.848)

\section{DENNSTAEDTIACEAE}

+ Pteridium aquilinum (L.) Kuhn subsp. aquilinum - G, Co - 4 (Sam.2)

DRYOPTERIDACEAE

+ Polystichum setiferum (Forssk.) Woynar - G, EA - 65 (Sam.942)

OPHIOGLOSSACEAE

+ Ophioglossum vulgatum L. - G, Ct - 64 (Sam.940)

\section{SPERMATOPHYTA - GYMNOSPERMAE}

\section{CUPRESSACEAE}

Juniperus oxycedrus subsp. deltoides (R. P. Adams) N. G. Passal. - P, EM - B\&Q (1976:15), 1 (Sam.24)

PINACEAE

+ - Abies cephalonica Loudon - P, Endem. - 4 (Sam.46), 11 (Sam.47), 93 (Sam.1072), 76 (Sam.1088)

\section{TAXACEAE}

+ Taxus baccata L. - P, EA - 99 (Sam.1067)

\section{SPERMATOPHYTA - ANGIOSPERMAE DICOTYLEDONEAE}

\section{ACANTHACEAE}

+ Acanthus spinosus L. - H, Me - obs.

ACERACEAE

+ Acer campestre L. - P, EA - 3 (Sam.70), 4 (Sam.71), 27 (Sam.648), 87 (Sam.1192)

+ Acer monspessulanum L. subsp. monspessulanum - P, ME - 1 (Sam.72), 3 (Sam.73), 10 (Sam.74), 88 (Sam.1203)

* Amaranthus caudatus L. - T, X - W\&W (2006:38) 
* Amaranthus hybridus L. - T, X - W\&W (2006:39)

* Amaranthus retroflexus L. - T, X - W\&W (2006:40)

\section{ANACARDIACEAE}

Cotinus coggygria Scop. - P, EA - B\&Q (1976:15), 95 (Sam.566)

Pistacia terebinthus L. subsp. terebinthus - P, Me - B\&Q (1976:15), 15 (Sam.411), 88 (Sam.1200), 95 (Sam.567)

\section{APIACEAE (UMBELLIFERAE)}

+ Anthriscus sylvestris subsp. nemorosus (M. Bieb.) Koso-Pol. - H, Pt - 12 (Sam.155), 65 (Sam.943)

+ Bunium ferulaceum Sm. - G, EM. - 2 (Sam.167)

+ Bupleurum glumaceum Sm. - T, Bk - 91 (Sam.1364)

+ Chaerophyllum nodosum (L.) Crantz $\{=$ Myrrhoides nodosa (L.) Cannon $\}-$ T, MS - 3 (Sam.170), 67 (Sam.957b)

* Daucus carota L. s.lat. - T H, Pt - W\&W (2006:62)

+ Dichoropetalum vittijugum (Boiss.) Pimenov \& Kljuykov $\{=$ Peucedanum vittijugum Boiss. $\}$ H, Bk - 7 (Sam.156), 10 (Sam.157), 28 (Sam.662), 88 (Sam.1199)

+ Eryngium campestre L. - H, EA - 13 (Sam.175), 15 (Sam.419)

+ Ferulago sylvatica (Besser) Rchb. subsp. sylvatica - H, BI - 53 (Sam.869)

* Foeniculum vulgare Mill. - H, Me - W\&W (2006:70) Geocaryum capillifolium (Guss.) Coss. - G, BI - B\&Q (1976:29), 2 (Sam.171), 3 (Sam.172), 4 (Sam.173), 12 (Sam.174), 16 (Sam.451), 17 (Sam.470), 24 (Sam.618)

+ Oenanthe pimpinelloides L. s.lat. - H, EA - 3 (Sam.168, Sam.169), 17 (Sam.469), 19 (Sam.506), 22 (Sam.585), 86 (Sam.1181)

+ Opopanax chironium (L.) W.D.J. Koch - H, ME - 88 (Sam.1205)

+ Orlaya daucoides (L.) Greuter - T, MS - 15 (Sam.409), 42 (Sam.774), 45 (Sam.814), 87 (Sam.1185), 89 (Sam.1219), 96 (Sam.550), 97 (Sam.1124)

* Physospermum cornubiense (L.) DC. - H, EA - B\&Q (1976:29)

* Pimpinella saxifraga L. - H, ES - W\&W (2008:125)

+ Scandix pecten-veneris L. - T, EA - 96 (Sam.549)

+ Selinum silaifolium (Jacq.) Beck $\{=$ Cnidium silaifolium (Jacq.) Simonkai $\}$ - H, ME - 4 (Sam.176), 13 (Sam.177), 18 (Sam.488), 24 (Sam.619), 27 (Sam.653)

+ Smyrnium perfoliatum subsp. rotundifolium (Mill.) Hartvig - H, Me - 3 (Sam.164, Sam.165), 10 (Sam.166)

+ Tordylium apulum L. - T, Me - 97 (Sam.1128), 98 (Sam.581)

* Tordylium maximum L. - T, EA - W\&W (2008:153) Torilis africana Spreng. $\{=$ T. arvensis subsp. purpurea (Ten.) Hayek $\}-\mathrm{T}$, ME - W\&W (2008:154), 1 (Sam.158), 2 (Sam.159), 3 (Sam.160, Sam.161), 6 (Sam.162), 12 (Sam.163), 16 (Sam.450), 56 (Sam.894), 85 (Sam.1154), 87 (Sam.1191)

+ Torilis leptophylla (L.) Rchb. f. - T, EA - 90 (Sam.1393)

\section{APOCYNACEAE}

+ Nerium oleander L. - $\mathrm{P}, \mathrm{Me}-\mathrm{obs}$.

+ Vinca major L. subsp. major - C, ME - obs.

AQUIFOLIACEAE

+ Ilex aquifolium L. - P, ME - 83 (Sam.1056) 
ARALIACEAE

+ Hedera helix L. subsp. helix - P, ME - 3 (Sam.75), 5 (Sam.76), 11 (Sam.77)

\section{ARISTOLOCHIACEAE}

+ Aristolochia elongata (Duch.) E. Nardi - G, Bk - 3 (Sam.3), 13 (Sam.4), 15 (Sam.435)

+ Aristolochia rotunda subsp. insularis (Nardi \& Arrigoni) Gamisans - G, ME - 3 (Sam.5)

ASTERACEAE (COMPOSITAE)

+ Achillea grandifolia Friv. - H, BA - 84 (Sam.1081)

+ Achillea nobilis subsp. neilreichii (A. Kern.) Velen. - H, EA - 90 (Sam.1394)

+ Aetheorhiza bulbosa (L.) Cass. s.lat. - G, Me - 15 (Sam.418)

+ Anthemis tinctoria subsp. parnassica (Boiss. \& Heldr.) Nyman - H, Bk - 24 (Sam.617)

* Anthemis arvensis L. subsp. arvensis - T, EA - W\&W (2006:43)

* Anthemis cotula L. - T, EA - W\&W (2006:43)

* Anthemis ruthenica M. Bieb. - T, EA - W\&W (2008:52)

+ Bellis annua L. subsp. annua - T, Me - 1 (Sam.260)

* Bellis perennis L. - H, EA - W\&W (2006:47)

* Carduus pycnocephalus L. - H, ME - W\&W (2008:64)

+ Carlina corymbosa subsp. graeca (Heldr. \& Sartori) Nyman - H, BA - 5 (Sam.267), 10 (Sam.268), 14 (Sam.269), 15 (Sam.425), 22 (Sam.595), 85 (Sam.1278), 88 (Sam.1196), 89 (Sam.1230), 54 (Sam.876)

+ Centaurea pichleri Boiss. - H, BA - 78 (Sam.1027)

* Centaurea salonitana Vis. - H, EA - W\&W (2008:67)

* Centaurea solstitialis L. subsp. solstitialis - H, Me - W\&W (2008:67)

* Chondrilla juncea L. - H, ME - W\&W (2006:55) Cichorium intybus L. - H, EA - W\&W (2008:72), 85 (Sam.1274)

* Cirsium ligulare subsp. albanum Wettst. - H, Bk - W\&W (2006:57)

+ Crepis fraasii Sch. Bip. subsp. fraasii - H, EM - 2 (Sam.272), 3 (Sam.273), 4 (Sam.274), 12 (Sam.275), 13 (Sam.276), 22 (Sam.601)

* Crepis neglecta L. s.lat. \{“Crepis capillaris (L.) Walk.”\} - T, BI - W\&W (2008:77)

* Crepis setosa Haller f. - T, EA - W\&W (2008:77)

+ Crupina crupinastrum (Moris) Vis. - T, EA - 90 (Sam.1402)

* Dittrichia graveolens (L.) Greuter - T, Me - W\&W (2006:64)

Doronicum orientale Hoffm. - G, EA - B\&Q (1976:29), 3 (Sam.277), 11 (Sam.278, Sam.279), 16 (Sam.456)

* Erigeron bonariensis L. \{= Conyza bonariensis (L.) Cronquist $\}$ - H, X - W\&W (2006:58)

* Erigeron canadensis L. \{= Conyza canadensis (L.) Cronquist $\}-$ H T, X - W\&W (2006:59)

* Filago germanica (L.) Huds. \{= Filago vulgaris Lam. $\}$ - T, Pt - W\&W (2008:86)

* Glebionis segetum (L.) Fourr. \{= Chrysanthemum segetum L. $\}$ - T, Me - W\&W (2008:72)

* Helminthotheca echioides (L .) Holub \{= Picris echioides L.\} - T, Me - W\&W (2008:124)

+ - Hieracium bracteolatum subsp. reinholdii (Boiss.) Zahn - H, Endem. - 24 (Sam.616), 35 (Sam.734), 41 (Sam.764), 48 (Sam.1280), 57 (Sam.899)

* Hypochaeris achyrophorus L. - T, Me - W\&W (2008:98)

* Hypochaeris radicata L. - H, EA - W\&W (2008:98)

+ Lactuca hispida DC. $\{=$ Cephalorrhynchus tuberosus (Steven) Schcian $\}$ - G, EA - 51 (Sam.850)

+ Lactuca muralis (L.) Gaertn. \{= Mycelis muralis (L.) Dumort. $-\mathrm{H}, \mathrm{Pt}$ - 11 (Sam.290) Lapsana communis subsp. adenophora (Boiss.) Rech. f. - H, BA - B\&Q (1976:29), 52 (Sam.856), 64 (Sam.937), 70 (Sam.969), 72 (Sam.979), 84 (Sam.1077) 
Leontodon tuberosus L. - H, Me - W\&W (2006:76), 1 (Sam.289), 15 (Sam.423), 19 (Sam.509), 31 (Sam.689), 88 (Sam.1202), 89 (Sam.1228)

Matricaria chamomilla L. $\{=$ Chamomilla recutita (L.) Rauschert\} - T, Co - W\&W (2008:71), obs.

+ Pilosella bauhini subsp. magyarica (Peter) S. Bräut. in Greuter \& Raus = Hieracium bauhini Schult.\} - H, EA - 3 (Sam.280), 12 (Sam.282), 76 (Sam.1003), 89 (Sam.1233)

+ Pilosella cymosa subsp. sabina (Sebast.) H.P. Fuchs - H, EA - 13 (Sam.285), 81 (Sam.1042)

+ Ptilostemon strictus (Ten.) Greuter in Greuter \& Rech. f. - H, BI - 11 (Sam.270), 35 (Sam.733), 42 (Sam.780), 48 (Sam.829, 1281)

+ Rhagadiolus stellatus (L.) Gaertn. - T, Me - 1 (Sam.291), 7 (Sam.292), 15 (Sam.422), 16 (Sam.454)

Scolymus hispanicus L. subsp. hispanicus - H, Me - W\&W (2006:91), obs.

+ Scorzoneroides cichoriacea (Ten.) Greuter in Greuter, Gutermann \& Talavera \{= Leontodon cichoriaceus (Ten.) Sanguin.\} - G, Me - 2 (Sam.286), 3 (Sam.287, Sam.288), 16 (Sam.455), 18 (Sam.490), 19 (Sam.510), 22 (Sam.600), 28 (Sam.1264), 85 (Sam.1172)

* Senecio vulgaris L. - T, Pt - W\&W (2006:91)

Sonchus asper (L.) Hill subsp. asper - T, Pt - W\&W (2008:148), 100 (Sam 568)

* Symphyotrichum squamatum (Spreng.) G.L. Nesom \{=Aster squamatus (Spreng.) Hieron. $\}$ - C $\mathrm{H}, \mathrm{X}-\mathrm{W} \& \mathrm{~W}(2006: 44)$

* Tanacetum parthenium (L.) Sch. Bip. - H, EA - W\&W (2006:98)

* Xanthium spinosum L. - T, X - W\&W (2006:104)

* Xanthium strumarium L. subsp. strumarium - T, Pt - W\&W (2006:104)

\section{BETULACEAE}

+ Carpinus orientalis Mill. subsp. orientalis - P, EA - 3 (Sam.6), 68 (Sam.960), 74 (Sam.985), 76 (Sam.1001)

+ Ostrya carpinifolia Scop. - P, MS - 41 (Sam.763), 64 (Sam.932), 81 (Sam.1083), 100 (Sam.571)

+ Alnus glutinosa (L.) Gaertn. subsp. glutinosa - P, ES - 130 (Sam.564)

+ Corylus avellana L. - P, EA - 64 (Sam.941)

\section{BORAGINACEAE}

+ - Alkanna calliensis Boiss.- H, Endem. - 91 (Sam.1378), 123 (Sam. 559)

+ Anchusa undulata subsp. hybrida (Ten.) Bég. - H, Me - 90 (Sam.1391)

+ Buglossoides purpurocaerulea (L.) I. M. Johnst. - H, EA - 3 (Sam.258, Sam.259), 15 (Sam.427), 102 (Sam.529)

Echium italicum subsp. biebersteinii (Lacaita) Greuter \& Burdet - H, MS - W\&W (2008:82), obs.

* Heliotropium europaeum L. - T, ME - W\&W (2006:71)

* Heliotropium hirsutissimum Grauer - T, EM - W\&W (2006:72)

+ Myosotis ramosissima Rochel subsp. ramosissima - T, EA - 1 (Sam.252), 2 (Sam.253), 3 (Sam.254), 15 (Sam.428), 30 (Sam.677), 31 (Sam.691), 32 (Sam.692), 42 (Sam.771), 78 (Sam.1024)

Myosotis sylvatica subsp. cyanea (Hayek) Vestergren - H T, Me - B\&Q (1976:29), 4 (Sam.255), 11 (Sam.256), 12 (Sam.257), 42 (Sam.778), 43 (Sam.788), 44 (Sam.795), 45 (Sam.816), 51 (Sam.851), 53 (Sam.870), 65 (Sam.944), 69 (Sam.964), 74 (Sam.988), 75 (Sam.991), 76 (Sam.999), 80 (Sam.1037), 81 (Sam.1044), 82 (Sam.1048), 83 (Sam.1064)

+ Neatostema apulum (L.) I. M. Johnst. - T, Me - obs. Symphytum bulbosum K. F. Schimp. - G, Me - B\&Q (1976:29), 1 (Sam.250), 13 (Sam.251)

\section{BRASSICACEAE (CRUCIFERAE)}

+ Aethionema saxatile subsp. graecum (Boiss. \& Spruner) Hayek - C, BA - 90 (Sam.1415) 
+ Alliaria petiolata (M. Bieb.) Cavara \& Grande - H, Pt - 70 (Sam.972)

+ Alyssum montanum subsp. repens (Baumg.) Schmalh. - C, ME - 90 (Sam.1416)

+ Alyssum simplex Rudolphi $\{=$ Alyssum minus (L.) Rothm. $\}$ - T, ES - 97 (Sam.1125), 125 (Sam.1137)

+ Arabis collina Ten. - H, Me - 73 (Sam.984)

+ Arabis glabra (L.) Bernh. - H, Eu - 91 (Sam.1365)

+ Arabis turrita L. - H, EA - 51 (Sam.849)

+ Arabis verna (L.) R. Br. in W. T. Aiton - T, Me - 79 (Sam.1250) Capsella bursa-pastoris (L.) Medik. - T H, Co - W\&W (2008:63), obs.

+ Cardamine bulbifera (L.) Crantz- G, Eu - 64 (Sam.939)

+ Cardamine graeca L. - T, Me - 2 (Sam.7), 14 (Sam.8)

+ Cardamine hirsuta L. - T, Co - 2 (Sam.9), 11 (Sam.10), 16 (Sam.463)

+ Draba muralis L. - T, ME - 85 (Sam.1163), 78 (Sam.1023)

+ • Erysimum asperulum Boiss. \& Heldr. in Boiss. - H, Endem. - 91 (Sam.1366)

+ Erysimum microstylum Hausskn. - H, Bk - 90 (Sam.1417)

+ Lepidium draba L. subsp. draba - G H, Co - 111 (Sam.575)

+ Nasturtium officinale $\mathrm{R}$. Br. - H, Co - 120 (Sam.533)

* Raphanus raphanistrum L. subsp. raphanistrum - T, EA - W\&W (2008:135) Rapistrum rugosum (L.) All. - T, EA - W\&W (2008:135), 103 (Sam.s.n.), 105 (Sam.538) Sisymbrium officinale (L.) Scop. - T, ES/[Co] - W\&W (2008:147), 105 (Sam.539)

\section{CAESALPINIACEAE}

Cercis siliquastrum L. - P, MS - B\&Q (1976:15), obs.

CAMPANULACEAE

* Campanula ramosissima Sm. - T, Me - W\&W (2008:63)

* Campanula sparsa subsp. sphaerothrix (Griseb.) Hayek - T, Bk - B\&Q (1976:29)

+ Campanula spatulata subsp. spruneriana (Hampe) Hayek - G, Bk - 1 (Sam.263), 2 (Sam.264), 3 (Sam.265), 12 (Sam.266), 44 (Sam.794), 84 (Sam.1080), 29 (Sam.1270) Campanula trachelium subsp. athoa (Boiss. \& Heldr.) Hayek - H, Bk - B\&Q (1976:29), 41 (Sam.765), 84 (Sam.1076)

+ Legousia speculum-veneris (L.) Chaix - T, ME - 90 (Sam.1405)

\section{CAPRIFOLIACEAE}

+ Lonicera etrusca Santi - P, Me - 21 (Sam.525), 3 (Sam.262)

* Lonicera implexa Aiton - P, Me - B\&Q (1976:15)

\section{CARYOPHYLLACEAE}

+ Arenaria serpyllifolia L. - T, EA - 14 (Sam.11)

+ Cerastium brachypetalum subsp. roeseri (Boiss. \& Heldr.) Nyman - T, Me - 31 (Sam.686), 32 (Sam.697), 54 (Sam.875), 77 (Sam.1012), 78 (Sam.1020)

+ Cerastium brachypetalum subsp. tenoreanum (Ser.) Soó - T, Eu - 16 (Sam.461), 28 (Sam.659), 85 (Sam.1174)

+ Cerastium glomeratum Thuill. - T, Co - 104 (Sam.376)

+ Dianthus viscidus Bory \& Chaub - H, BA - 7 (Sam.398)

+ Petrorhagia illyrica (L.) P. W. Ball \& Heywood subsp. illyrica - H, Bk - 91 (Sam.1375)

+ Minuartia recurva subsp. condensata (C. Presl) Greuter \& Burdet - H C, ME - 90 (Sam.1418)

+ Minuartia hybrida (Vill.) Schischk in Kom. - T, EA - 90 (Sam.1420)

+ Moehringia pentandra J. Gay - T, Me - 67 (Sam.958) 
+ Moenchia mantica (L.) Bartl. - T, ME - 31 (Sam.685), 54 (Sam.874), 105 (Sam.546)

+ Petrorhagia dubia (Raf.) G. López \& Romo - T, Me - 90 (Sam.1419), 105 (Sam.544)

+ Petrorhagia obcordata (Margot \& Reut.) Greuter \& Burdet - T, BA - 91 (Sam.1371)

+ Lychnis coronaria (L.) Desr. \{= Silene coronaria (L.) Clairv. $\}$ - H, MS - 35 (Sam.729)

+ Silene conica L. - T, EA - 90 (Sam.1422)

+ Silene cretica L. - T, Me - 105 (Sam.545) Silene italica (L.) Pers. subsp. italica - H, EA - B\&Q (1976:29), 2 (Sam.12), 4 (Sam.14), 12 (Sam.13), 28 (Sam.1259)

+ Silene multicaulis Guss. subsp. multicaulis - H, BI - 84 (Sam.1073)

+ Silene viridiflora L. - H, ES - 56 (Sam.892)

+ Silene vulgaris subsp. bosniaca (Beck) Greuter, Burdet \& Long - H, Me - 12 (Sam.15)

+ Stellaria cupaniana Jord. \& Fourr. - T, Me - 1 (Sam.20), 2 (Sam.21), 15 (Sam.433), 16 (Sam.460), 17 (Sam.478), 18 (Sam.496), 28 (Sam.1257), 87 (Sam.1183), 89 (Sam.1221)

+ Stellaria media (L.) Vill. - T H, Co - 3 (Sam.17), 4 (Sam.18), 11 (Sam.19), 18 (Sam.495), 43 (Sam.785a), 45 (Sam.817), 53 (Sam.867), 78 (Sam.1245), 79 (Sam. 1249), 85 (Sam.1168), 87 (Sam.1187), 16 (Sam. 1241)

+ Velezia rigida L. - T, MS - 91 (Sam.1380)

\section{CHENOPODIACEAE}

* Atriplex patula L. - T, ES - W\&W (2006:45)

* Chenopodium album L. - T, Co - W\&W (2008:71)

* Chenopodium opulifolium Sehrad. - T, EA - W\&W (2006:53)

* Chenopodium vulvaria L. - T, EA - W\&W (2006:54)

* Dysphania ambrosioides (L.) Mosyakin \& Clemants $\{=$ Chenopodium ambrosioides L. $\}$ - T H, X - W\&W (2006:52)

* Dysphania botrys (L.) Mosyakin \& Clemants $\{=$ Chenopodium botrys L. $\}$ - T, EA - W\&W (2006:53)

\section{CISTACEAE}

Cistus creticus L. subsp. creticus - C, Me - B\&Q (1976:15), 5 (Sam.78), 19 (Sam.507)

+ Cistus salviifolius L. - C, Me - 106 (Sam.565)

+ Helianthemum nummularium (L.) Mill. subsp. nummularium - C, Me - 107 (Sam.1099)

+ Tuberaria guttata (L.) Fourr. - T, MA - 113 (Sam.1208)

\section{CONVOLVULACEAE}

Calystegia silvatica (Kit.) Griseb. - H, MS - W\&W (2008:62), obs.

+ Convolvulus arvensis L. - H G, Co - 90 (Sam.1388)

+ Cuscuta brevistyla A. Rich. - T, ST - 42 (Sam.773)

\section{CORNACEAE}

+ Cornus mas L. - P, EA - 3 (Sam.79)

\section{CRASSULACEAE}

+ Sedum amplexicaule subsp. tenuifolium (Sm.) Greuter - C H, Me - 14 (Sam.22), 25 (Sam.630), 52 (Sam.863), 90 (Sam.1382)

+ Sedum cepaea L. - T, Me - 30 (Sam.682), 35 (Sam.739), 44 (Sam.800), 57 (Sam.898)

+ Sedum hispanicum L. - H C, EA - 90 (Sam.1384)

+ Umbilicus luteus (Huds.) Webb \& Berthel. - G, Me - 82 (Sam.1051) 
CUCURBITACEAE

* Ecballium elaterium (L.) A. Rich. - G, MS - W\&W (2006:65)

\section{DIPSACACEAE}

* Knautia integrifolia (L.) Bertol. subsp. integrifolia - T, Me - W\&W (2008:99)

+ Pterocephalus plumosus (L.) Coult. - T, MS - 91 (Sam.1367)

* Scabiosa columbaria L. subsp. columbaria - H, EA - W\&W (2008:141)

* Scabiosa atropurpurea L. \{= Sixalix atropurpurea (L .) Greuter \& Burdet $\}-$ H, Me - W\&W (2006:93)

\section{ERICACEAE}

+ Hypopitys monotropa Crantz subsp. monotropa $\{=$ Monotropa hypopitys L. $\}$ - G, Bo - 41 (Sam.769)

\section{EUPHORBIACEAE}

+ Euphorbia apios L. - G, Me - 3 (Sam.80, Sam.81), 24 (Sam.622)

+ Euphorbia peplus L. - T, Co - 15 (Sam.410), 88 (Sam.1197)

+ Euphorbia phymatosperma subsp. cernua (Coss. \& Durieu) Vindt - T, Me - 17 (Sam.468), 32 (Sam.698), 36 (Sam.742), 56 (Sam.893)

* Euphorbia prostrata Aiton - T, X - W\&W (2006:69)

\section{FABACEAE}

+ Astragalus glycyphyllos subsp. glycyphylloides (DC.) Maire \& Petitm. - C, BA - 44 (Sam.849)

+ Astragalus pelecinus (L.) Barneby - T, Me - 125 (Sam.1149)

+ Bituminaria bituminosa (L.) C.H. Stirt. - H, ME - 97 (Sam.1129) Chamaecytisus hirsutus (L.) Link subsp. hirsutus $\{=$ C. triflorus (Lam.) Skalická $\}-$ C, Eu B\&Q (1976:29), 7 (Sam.97), 35 (Sam.725)

+ Colutea arborescens L. subsp. arborescens - P, EA - 100 (Sam.572)

+ Dorycnium herbaceum Vill. - H, ME - 49 (Sam.840) Dorycnium hirsutum (L.) Ser. - H C, Me - B\&Q (1976:15), 22 (Sam.599), 23 (Sam.609)

+ Genista tinctoria L. subsp. tinctoria - C, ES - 24 (Sam.615), 30 (Sam.674), 62 (Sam.922), 111 (Sam.574)

+ Hippocrepis emerus subsp. emeroides (Boiss. \& Spruner) Greuter \& Burdet ex Lassen \{= Coronilla emerus subsp. emeroides (Boiss. \& Spruner) Lassen\} - P, EM - 27 (Sam.650, 59 (Sam.906, 100 (Sam.569

+ Hymenocarpos circinnatus (L.) Savi - T, Me - 97 (Sam.1126)

+ Lathyrus aphaca L. - T, MS - 1 (Sam.98), 3 (Sam.99), 18 (Sam.551)

+ Lathyrus cicera L. - T, MS - 103 (Sam.s.n.), 116 (Sam.1130)

+ Lathyrus digitatus (M. Bieb.) Fiori in Fiori \& Paol. - H, Me - 3 (Sam.100), 16 (Sam.449), 61 (Sam.918), 62 (Sam.920), 73 (Sam.982)

+ Lathyrus grandiflorus Sm. - H, BI - 76 (Sam.1006) Lathyrus laxiflorus (Desf.) Kuntze - H, EM - B\&Q (1976:29), 2 (Sam.101), 3 (Sam.102), 4 (Sam.103)

+ Lathyrus niger (L.) Bernh. - H, ME - 11 (Sam.148), 25 (Sam.635), 35 (Sam.728), 44 (Sam.789), 44 (Sam.789)

+ Lathyrus nissolia L. - T, ME - 21 (Sam.523)

+ Lathyrus sphaericus Retz. - T, EA - 2 (Sam.104), 3 (Sam.105), 10 (Sam.106), 15 (Sam.891), 56 (Sam.408)

+ Lathyrus venetus (Mill.) Wohlf. - H, Eu - 35 (Sam.726) 
+ Lens ervoides (Brign.) Grande - T, Me - 107 (Sam.1100)

+ Lotus conimbricensis Brot. - T, Me - 125 (Sam.1148)

+ Lotus corniculatus L. - H, EA - 117 (Sam.707)

+ Medicago arabica (L.) Huds. - T, EA - 1 (Sam.107)

+ Medicago coronata (L.) Bartal. - T, MS - 112 (Sam.1109)

+ Medicago disciformis DC. - T, Me - 97 (Sam.1119)

+ Medicago minima (L.) Bartal. - T, Pt - 97 (Sam.1116, 1118), 107 (Sam.1102)

+ Medicago orbicularis (L.) Bartal. - T, MS - 97 (Sam.1115)

+ Medicago polymorpha L. - T, Pt - 97 (Sam.1114)

* Medicago sativa L. subsp. sativa - H, EA - W\&W (2006:78)

+ Onobrychis caput-galli (L.) Lam. - T, Me - 97 (Sam.1117), 98 (Sam.580) Ornithopus compressus L. - T, Me - W\&W (2008:120), 21 (Sam.522, Sam.555)

+ Robinia pseudoacacia L. - P, X - obs.

+ Scorpiurus muricatus L. - T, Me - 97 (Sam.1127)

+ Securigera varia (L.) Lassen $\{=$ Coronilla varia L. $\}$ - H, EA - 81 (Sam.1084)

+ Spartium junceum L. - P, Me - obs.

+ Trifolium angustifolium L. - T, EA - 10 (Sam.108), 21 (Sam.521), 29 (Sam.670) Trifolium arvense L. - T, Pt - W\&W (2008:156), 31 (Sam.683), 24 (Sam.612), 110 (Sam.577)

+ Trifolium campestre Schreb. - T, EA - 3 (Sam.109), 22 (Sam.594), 29 (Sam.667), 32 (Sam.701), 57 (Sam.897), 125 (Sam.1136)

+ Trifolium grandiflorum Schreb. - T, MS - 1 (Sam.127), 2 (Sam.128), 12 (Sam.129), 24 (Sam.611), 25 (Sam.631), 28 (Sam.657, Sam.1261), 29 (Sam.668), 85 (Sam.1158), 111 (Sam.573)

+ Trifolium heldreichianum Hausskn. - G, BA - 7 (Sam.110, Sam.399), 25 (Sam.632), 32 (Sam.695), 33 (Sam.712)

+ Trifolium hirtum All. - T, Me - 90 (Sam. 1381)

+ Trifolium medium subsp. balcanicum Velen. - G, Bk - 14 (Sam.131), 44 (Sam.793), 47 (Sam.823), 49 (Sam.833), 50 (Sam.839), 55 (Sam.885a), 71 (Sam.974), 74 (Sam.987), 80 (Sam.1036), 81 (Sam.1043), 84 (Sam.1078)

* Trifolium nigrescens Viv. - T, Me - W\&W (2006:100)

Trifolium ochroleucon subsp. roseum (C. Presl) Lassen in Greuter \& Raus - H, Me - B\&Q (1976:29), 2 (Sam.112), 3 (Sam.113), 12 (Sam.114), 16 (Sam.448), 19 (Sam.502), 23 (Sam.604), 25 (Sam.633), 29 (Sam.671), 30 (Sam.673), 47 (Sam.824), 48 (Sam.827), 55 (Sam.885b), 83 (Sam.1061)

+ Trifolium pannonicum Jacq. - G, ME - 67 (Sam.956), 78 (Sam.1026)

+ Trifolium patulum Tausch - G, BI - 24 (Sam.614), 35 (Sam.724), 36 (Sam.743)

Trifolium physodes M. Bieb. - H, Me - B\&Q (1976:29), 1 (Sam.122), 2 (Sam.123), 3 (Sam.124), 12 (Sam.125), 14 (Sam.126), 26 (Sam.642)

Trifolium pignantii Fauché \& Chaub. - G, Bk - B\&Q (1976:29), 2 (Sam.115), 4 (Sam.116), 6 (Sam.117), 7 (Sam.118), 11 (Sam.119), 12 (Sam.120), 14 (Sam.121), 27 (Sam.649), 32 (Sam.694), 33 (Sam.713), 85 (Sam.1164), 111 (Sam.576)

* Trifolium pratense L. - H, EA - W\&W (2006:100)

* Trifolium repens $\mathrm{L}$. - H, Pt - W\&W (2006:101)

+ Trifolium scabrum L. - T, EA - 91 (Sam.1374), 97 (Sam.1113)

+ Trifolium stellatum L. - T, Me - 19 (Sam.505), 89 (Sam.1215), 112 (Sam.1108), 21 (Sam.556)

+ Trifolium subterraneum L. - T, ME - 1 (Sam.130), 19 (Sam.504), 21 (Sam.520), 114 (Sam.1104)

+ Trifolium tenuifolium Ten. - T, EM - 24 (Sam.613), 29 (Sam.669), 42 (Sam.775), 54 (Sam.873)

+ Trifolium tomentosum L. - T, Me - 125 (Sam.1147)

* Trigonella corniculata (L.) L. subsp. corniculata - T, Me - W\&W (2006:101) 
+ Tripodion tetraphyllum (L.) Fourr. $\{=$ Anthyllis tetraphylla L. $\}-$ T, Me - 98 (Sam.582)

*Vicia angustifolia L. \{=Vicia sativa (L.) Ehrh. subsp. nigra $\}$ - T, Pt - W\&W (2006:103)

+ Vicia bithynica (L.) L. - T, Me - 113 (Sam.1211)

+ Vicia cassubica L. - H, EA - 4 (Sam.147), 18 (Sam.487), 33 (Sam.714), 35 (Sam.727), 38 (Sam.748)

* Vicia cracca L. - H, Ct - W\&W (2006:103) Vicia grandiflora Scop. - T, ME - W\&W (2008:168), 2 (Sam.132), 3 (Sam.133, Sam.134), 7 (Sam.136, Sam.137), 10 (Sam.138), 26 (Sam.643), 28 (Sam.1258), 30 (Sam.675), 31 (Sam.684), 32 (Sam.696, Sam.700), 41 (Sam.766), 42 (Sam.772, Sam.779), 45 (Sam.813), 67 (Sam.957a), 117 (Sam.708)

+ Vicia hirsuta (L.) Gray - T, Pt - 2 (Sam.139), 3 (Sam.140, Sam.141), 10 (Sam.142), 15 (Sam.404), 16 (Sam.447), 18 (Sam.486), 19 (Sam.499), 21 (Sam.518)

+ Vicia incisa M. Bieb. \{=V. sativa L. subsp. incisa (M.Bieb.) Arcang. - T, EM - 2 (Sam.145), 3 (Sam.146), 32 (Sam.693)

+ Vicia lathyroides L. - T, ME - 1 (Sam.143), 3 (Sam.144), 13 (Sam.149), 15 (Sam.407), 21 (Sam.519b), 38 (Sam.747), 44 (Sam.790), 54 (Sam.871), 57 (Sam.896), 76 (Sam.1005), 77 (Sam.1010), 78 (Sam.1097), 79 (Sam.1251), 103 (Sam.s.n.), 114 (Sam.1105)

+ Vicia pannonica subsp. striata (M. Bieb) Nyman - T, ME - 103 (Sam.s.n.)

+ Vicia pubescens (DC.) Link - T, Me - 15 (Sam.405), 89 (Sam.1216)

+ Vicia tenuifolia subsp. dalmatica (A. Kern.) Greuter in Greuter \& Raus - H, ME - 19 (Sam.500)

+ Vicia tetrasperma (L.) Shreb. - T, Pt - 17 (Sam.406), 19 (Sam.501), 29 (Sam.666), 34 (Sam.720) Vicia villosa subsp. varia (Host) Corb. - T, ME - B\&Q (1976:29), 105 (Sam.541)

\section{FAGACEAE}

Castanea sativa Mill. - P, Eu - B\&Q (1976:29), 81 (Sam.1040), 82 (Sam.1052)

+ Quercus coccifera L. - P, Me - 4 (Sam.25)

Quercus frainetto Ten. - P, BA - B\&Q (1976:29), 1 (Sam.26), 3 (Sam.34), 4 (Sam.37), 5 (Sam.35, Sam.36b), 7 (Sam.28), 11 (Sam.27, Sam.39), 14 (Sam.44), 24 (Sam.621), 25 (Sam.628), 26 (Sam.638), 27 (Sam.647), 89 (Sam.1212)

+ Quercus petraea subsp. polycarpa (Schur) Soó - P, Eu - 4 (Sam.29), 11 (Sam.30), 12 (Sam.31), 13 (Sam.32), 26 (Sam.639), 80 (Sam.1034, Sam.1089), 93 (Sam.1071), 115 (Sam.1086)

Quercus pubescens Willd. - P, ME - B\&Q (1976:15), 1 (Sam.33), 5 (Sam.36a), 4 (Sam.38), 11 (Sam.40), 14 (Sam.41, Sam.43, Sam.45)

\section{FUMARIACEAE}

+ Corydalis cava (L.) Schweigger \& Körte - G, EA - 78 (Sam.1094)

+ Fumaria officinalis L. subsp. officinalis - T, Pt - 105 (Sam.543)

\section{GENTIANACEAE}

+ Blackstonia perfoliata (L.) Huds. subsp. perfoliata - T, ME - obs.

+ Centaurium erythraea Rafn s.lat. - T H, EA - 24 (Sam.623, Sam.624)

\section{GERANIACEAE}

+ Erodium cicutarium (L.) L' Hér. in Aiton - T, Ct - 90 (Sam.1397), 116 (Sam.1131)

+ Geranium asphodeloides Burm. f. subsp. asphodeloides - H, MS - 3 (Sam.82, Sam.83, Sam.84), 49 (Sam.841), 52 (Sam.855)

+ Geranium columbinum L. - T, EA - 1 (Sam.85), 117 (Sam.710)

+ Geranium dissectum L. - T, EA - 21 (Sam.553)

Geranium lucidum L. - T, EA - W\&W (2008:91), 1 (Sam.86), 12 (Sam.87), 44 (Sam.799) 
+ Geranium peloponesiacum Boiss. - H, Bk - 119 (Sam.530), 18 (Sam.489), 28 (Sam.1256), 39 (Sam.755), 44 (Sam.798), 52 (Sam.854), 79 (Sam.1030)

+ Geranium pusillum Burm. f. - T, EA - 15 (Sam.413)

+ Geranium molle L. - T, Pt - 1 (Sam.88)

+ Geranium purpureum Vill. - T, Me - 1 (Sam.89), 3 (Sam.90), 13 (Sam.91), 14 (Sam.92), 15 (Sam.414), 16 (Sam.453), 34 (Sam.721), 85 (Sam.1161), 89 (Sam.1231)

+ Geranium rotundifolium L. - T, Pt - 1 (Sam.93)

HYPERICACEAE (GUTTIFERAE)

+ Hypericum perfoliatum L. - H, Me - 23 (Sam.606) Hypericum perforatum L. subsp. veronense (Schrank) A. Fröhl. - H, Pt - W\&W (2006:73), 7 (Sam.397)

+ Hypericum spruneri Boiss. - H, BI - 2 (Sam.95), 3 (Sam.96), 4 (Sam.94), 15 (Sam.412), 23 (Sam.605), 24 (Sam.620), 26 (Sam.641), 30 (Sam.676), 52 (Sam.862), 62 (Sam.923), 66 (Sam.950), 77 (Sam.1013)

\section{JUGLANDACEAE}

+ Juglans regia $\mathrm{L}$. - $\mathrm{P}, \mathrm{ME}$ - obs.

\section{LAMIACEAE (LABIATAE)}

+ Ajuga reptans L. - G, EA - 64 (Sam.936), 120 (Sam.532)

* Ballota nigra L. - H, EA - W\&W (2006:46)

+ Clinopodium alpinum subsp. meridionale (Nyman) Govaerts $\{=$ Acinos alpinus subsp. meridionalis (Nyman) P.W. Ball; Satureja alpina subsp. meridionalis (Nyman) Greuter \& Burdet $\}$ - H, Me - 90 (Sam.1395)

+ Clinopodium grandiflorum (L.) Kuntze $\{=$ Calamintha grandiflora (L.) Moench; Satureja grandiflora (L.) Scheele\} - H, ME - 53 (Sam.865)

* Clinopodium nepeta subsp. glandulosum (Req.) Govaerts \{= Calamintha nepeta subsp. glandulosa (Req.) P.W. Ball \}- H, ME - W\&W (2008:62)

Clinopodium vulgare subsp. orientale Bothmer $\{=$ Satureja vulgaris subsp. orientalis (Bothmer) Greuter \& Burdet, "C. vulgare subsp. arundanum (Boiss.) Nyman"\} - H, Me - B\&Q (1976:29), 91 (Sam.1379), 3 (Sam.239), 4 (Sam.238), 15 (Sam.429), 17 (Sam.474), 19 (Sam.512), 22 (Sam.593), 83 (Sam.1060)

+ Lamium bifidum subsp. balcanicum Velen. - T, Bk - 1 (Sam.246), 3 (Sam.247), 79 (Sam.1254), 87 (Sam.1186)

+ Lamium bifidum Cirillo subsp. bifidum - T, Me - 104 (Sam.390)

+ Lamium garganicum L. subsp. garganicum - H, Me - 14 (Sam.248, Sam.249), 55 (Sam.886), 65 (Sam.946), 82 (Sam.1049), 84 (Sam.1079), 122 (Sam.1098)

+ Lamium purpureum L. - T, EA - 104 (Sam.395)

* Marrubium peregrinum L. - H, Eu - W\&W (2006:77)

+ Melissa officinalis L. subsp. officinalis - H, Me - 132 (Sam.1032)

* Melittis melissophyllum subsp. albida (Guss.) P.W. Ball - H, Eu - B\&Q (1976:29)

* Mentha spicata subsp. condensata (Briq.) Greuter \& Burdet $\{=$ Mentha microphylla K. Koch $\}$ $\mathrm{H}, \mathrm{Me}-\mathrm{W} \& \mathrm{~W}(2006: 78)$

* Mentha pulegium L. - H, Me - W\&W (2006:79)

* Micromeria graeca (L.) Rchb. subsp. graeca - C, Me - B\&Q (1976:15)

+ Origanum vulgare subsp. hirtum (Link) A. Terracc. - H G, BA - 1 (Sam.245), 16 (Sam.458), 21 (Sam.526), 22 (Sam.592), 52 (Sam.859)

+ Phlomis fruticosa L. - P, Me - 1 (Sam.244) 
+ Prunella vulgaris L. - H, EA - 54 (Sam.877)

+ Salvia argentea L. - H, Me - 91 (Sam.1363)

+ Scutellaria columnae All. subsp. columnae - H, Me - 26 (Sam.645), 18 (Sam.491), 53 (Sam.866)

+ Stachys germanica subsp. heldreichii (Boiss.) Hayek - H, EM - 90 (Sam.1390)

+ - Stachys graeca Boiss. \& Heldr. in Boiss. \{"Stachys acutifolia Bory \& Chaub."\} - H, Endem. 15 (Sam.437), 20 (Sam.516), 117 (Sam.711)

* Teucrium capitatum L. subsp. capitatum - C, Me - B\&Q (1976:15)

+ Teucrium chamaedrys L. subsp. chamaedrys - C, Me - 16 (Sam.464), 22 (Sam.588), 85 (Sam.1173), 91 (Sam.1377)

* Teucrium divaricatum Heldr. s.lat. - C, EM - B\&Q (1976:15)

Thymus longicaulis subsp. chaubardii (Rchb. f.) Jalas \{= Thymus ocheus Boiss. - C, BA B\&Q (1976:29), 1 (Sam.240), 3 (Sam.241, Sam.242), 5 (Sam.243), 25 (Sam.636), 26 (Sam.644), 42 (Sam.776), 52 (Sam.858), 59 (Sam.908), 80 (Sam.1038), 90 (Sam.1385)

LORANTHACEAE

+ Loranthus europaeus Jacq. - P, EA - obs.

MALVACEAE

* Abutilon theophrastii Medik. - T, EA - W\&W (2006:36)

* Malva sylvestris L. - T H, EA - W\&W (2006:77)

MORINACEAE

+ Morina persica L. - H, MS - obs.

OLEACEAE

+ Fraxinus angustifolia subsp. oxycarpa (Willd.) Franco \& Rocha Afonso - P, EA - 4 (Sam.231), 11 (Sam.232), 14 (Sam.233, Sam.234, Sam.235)

+ Fraxinus ornus L. - P, ME - 3 (Sam.236), 14 (Sam.237), 44 (Sam.792)

Phillyrea latifolia L. - P, Me - B\&Q (1976:15), 3 (Sam.230)

ONAGRACEAE

* Epilobium hirsutum L. - H, EA - W\&W (2006:67)

+ Epilobium lanceolatum Sebast. \& Mauri - H, EA - 65 (Sam.947), 45 (Sam.812), 69 (Sam.965), 77 (Sam.1011)

PAPAVERACEAE

Papaver rhoeas L. - T, Pt - W\&W (2008:121), obs.

PLANTAGINACEAE

* Plantago major L. subsp. major - H, EA - W\&W (2008:127)

PLATANACEAE

+ Platanus orientalis L. - P, EM - obs.

PLUMBAGINACEAE

* Plumbago europaea L. - C P, Me - W\&W (2006:84)

POLYGONACEAE

* Persicaria maculosa Gray - T, EA - W\&W (2008:122) 
* Polygonum arenarium Waldst. \& Kit. - T, EA - W\&W (2006:84)

* Polygonum arenastrum Boreau - T, EA - W\&W (2006:85)

* Polygonum aviculare L. subsp. aviculare - T, Ct - W\&W (2006:85)

* Rumex pulcher L. subsp. pulcher - H, MS - W\&W (2008:138)

+ Rumex tuberosus L. subsp. tuberosus - G, MS - 13 (Sam.48), 39 (Sam.752), 76 (Sam.1000)

+ Rumex acetosella subsp. acetoselloides (Balansa) Nijs - H, EA - 90 (Sam.1383)

PRIMULACEAE

* Cyclamen graecum Link subsp. graecum - G, EM - W\&W (2006:61)

+ Cyclamen hederifolium Aiton subsp. hederifolium - G, Me - 1 (Sam.228), 3 (Sam.229), 44 (Sam.802), 85 (Sam.1275), 125 (Sam.1273)

+ Lysimachia atropurpurea L. - G, BA - 123 (Sam.562)

+ Lysimachia punctata L. - H, Eu - 35 (Sam.740), 64 (Sam.935)

\section{PUNICACEAE}

* Punica granatum L. - P, X - W\&W (2008:132)

\section{RANUNCULACEAE}

+ Anemone apennina subsp. blanda (Schott \& Kotschy) Nyman - G, EM - 3 (Sam.49, Sam.50), 45 (Sam.811), 52 (Sam.857), 64 (Sam.933)

+ Anemone pavonina Lam. - G, Me - 1 (Sam.51), 3 (Sam.52, Sam.53)

+ Clematis flammula L. - P, MS - 3 (Sam.54), 10 (Sam.55), 21 (Sam.527)

* Clematis vitalba L. - P, EA - W\&W (2006:57)

+ Consolida ajacis (L.) Schur \{'Consolida ambigua”\} - T, Me - 91 (Sam.1370)

* Delphinium peregrinum L. - T, MS - W\&W (2006:63)

+ Ficaria subsp. ficariiformis (F. W. Schultz) B. Walln. $\{=$ Ranunculus ficaria subsp. ficariiformis (F. W. Schultz) Rouy \& Fouc. - G, Me - 1 (Sam.62), 2 (Sam.63)

Helleborus odorus subsp. cyclophyllus (A. Braun) Maire \& Petitm. - G, Bk - B\&Q (1976:29), 3 (Sam.56, Sam.57), 4 (Sam.58), 11 (Sam.59), 35 (Sam.732)

Nigella damascena L. - T, Me - W\&W (2008:116), 90 (Sam.1400), 91 (Sam.1369)

+ Ranunculus gracilis E. D. Clarke - H, BA - 18 (Sam.494), 120 (Sam.535), 16 (Sam.1093)

+ Ranunculus neapolitanus Ten. $\{=$ R. bulbosus subsp. neapolitanus (Ten.) H. Lindb., "R. bulbosus subsp. aleae (Willk.) Rouy \& Fouc."\} - H, Me - 1 (Sam.64), 3 (Sam.65, Sam.66), 6 (Sam.67), 15 (Sam.432), 120 (Sam.534a)

+ Ranunculus paludosus Poir. - H, ME - 3 (Sam.60, Sam.61), 15 (Sam.431), 19 (Sam.513), 85 (Sam.1153), 89 (Sam.1223)

+ Ranunculus psilostachys Griseb. - H, Bk - 7 (Sam.68), 17 (Sam.476), 18 (Sam.493), 85 (Sam.1160a), 16 (Sam.1239)

+ Ranunculus velutinus Ten. - H, Me - 17 (Sam.477), 43 (Sam.786), 78 (Sam.1025), 120 (Sam.534b)

\section{RHAMNACEAE}

Paliurus spina-christi Mill. - P, EA - B\&Q (1976:15), 85 (Sam.1160b)

\section{ROSACEAE}

+ Agrimonia eupatoria L. subsp. eupatoria - H, EA - 3 (Sam.215), 1 (Sam.214), 15 (Sam.416), 22 (Sam.591)

Aremonia agrimonoides (L.) DC. subsp. agrimonoides - H, BC - B\&Q (1976:29), 1 (Sam.209), 3 (Sam.210, Sam.211, Sam.213), 4 (Sam.212) 
+ Crataegus monogyna Jacq. - P, Pt - 1 (Sam.204), 10 (Sam.205), 11 (Sam.206), 13 (Sam.207), 14 (Sam.208), 17 (Sam.472)

+ Geum urbanum L. - H, EA - 69 (Sam.967), 124 (Sam.1096)

+ Malus sylvestris (L.) Mill. subsp. sylvestris - P, Eu - 81 (Sam.1085)

+ Potentilla inclinata Vill. - H, EA - 35 (Sam.741), 90 (Sam.1398) Potentilla micrantha DC. in Lam. \& DC. - H, EA - B\&Q (1976:29), 3 (Sam.199, Sam.200), 4 (Sam.201), 11 (Sam.202), 27 (Sam.651), 33 (Sam.715), 39 (Sam.754), 40 (Sam.760), 51 (Sam.853), 52 (Sam.861), 83 (Sam.1068)

+ Prunus avium (L.) L. - P, EA - 49 (Sam.842), 69 (Sam.962), 70 (Sam.968), 84 (Sam.1074) Prunus cocomilia Ten. - P, EM - B\&Q (1976:15), obs.

+ Prunus domestica subsp. insititia (L.) Bonnier \& Layens - P, EA - 3 (Sam.190), 7 (Sam.191), 11 (Sam.192, Sam.193), 13 (Sam.194), 14 (Sam.195), 17 (Sam.471), 26 (Sam.640), 34 (Sam.723), 35 (Sam.731), 52 (Sam.860), 64 (Sam.931), 65 (Sam.945), 66 (Sam.948), 76 (Sam.1002), 84 (Sam.1075)

+ Prunus mahaleb L. subsp. mahaleb - P, EA - 4 (Sam.196), 11 (Sam.197, Sam.198), 44 (Sam.791), 49 (Sam.843), 55 (Sam.884)

* Pyrus communis L. - P, EA - B\&Q (1976:29)

+ Pyrus spinosa Forssk. \{= P. amygdaliformis Vill.\} - P, Me - 15 (Sam.417), 74 (Sam.986)

+ Rosa arvensis Huds. - P, Me - 3 (Sam.182, 183, 401, 402, 403), 4 (Sam.184), 7 (Sam.400), 21 (Sam.524), 45 (Sam.807a), 64 (Sam.929b), 66 (Sam.949), 82 (Sam.1054), 85 (Sam.1166), 88 (Sam.1194), 89 (Sam.1224)

+ Rosa canina L. - P, Pt - 10 (Sam.186), 45 (Sam.807b), 56 (Sam.889), 59 (Sam.905), 64 (Sam.930)

+ Rosa pulverulenta M. Bieb. $\{=$ R. glutinosa Sm. $\}$ - P, ME - 4 (Sam.187), 11 (Sam.189)

+ Rosa tomentosa Sm. in Sm. \& Sowerby - C, ME - 64 (Sam.929a), 70 (Sam.970), 82 (Sam.1055), 86 (Sam.1179)

+ Rubus canescens DC. - P, EA - 1 (Sam.178), 2 (Sam.179), 3 (Sam.180), 4 (Sam.181), 41 (Sam.762), 64 (Sam.926), 70 (Sam.971), 80 (Sam.1035), 83 (Sam.1069)

+ Rubus hirtus Waldst. \& Kit. - P, EA - 64 (Sam.927), 81 (Sam.1041), 83 (Sam.1058, Sam.1070) Rubus sanctus Schreb. $\{=$ R. ulmifolius Schott $\}$ - P, MS - W\&W (2006:88), 64 (Sam.928)

+ Sanguisorba minor subsp. balearica (Nyman) Muños Garm. \& C. Navaro $\{=$ S. minor subsp. muricata (Bonnier \& Layens) Briq.\} - H, EA - 15 (Sam.415), 22 (Sam.597), 100 (Sam.570), 123 (Sam.560)

Sorbus torminalis (L.) Crantz - P, EA - B\&Q (1976:29), 25 (Sam.629), 58 (Sam.904)

+ Sorbus umbellata (Desf.) Fritsch in A. Kern. - P, BA - 83 (Sam.1059)

\section{RUBIACEAE}

+ Crucianella angustifolia L. - T, ME - 7 (Sam.296), 91 (Sam.1376)

+ Cruciata pedemontana (Bellardi) Ehrend. - T, EA - 7 (Sam.295)

+ Galium aparine L. - T, EA - 1 (Sam.297), 2 (Sam.298), 3 (Sam.299), 11 (Sam.300), 41 (Sam.768), 45 (Sam.810)

+ Galium heldreichii Halácsy - H, BA - 16 (Sam.457), 17 (Sam.473), 19 (Sam.511), 22 (Sam.589), 25 (Sam.634), 27 (Sam.655), 30 (Sam.680), 31 (Sam.687), 45 (Sam.808), 48 (Sam.828), 49 (Sam.845), 50 (Sam.838), 60 (Sam.912), 72 (Sam.978b), 85 (Sam.1171), 88 (Sam.1193), 89 (Sam.1226)

+ Galium hellenicum Krendl - H, Bk - 22 (Sam.590), 27 (Sam.654), 31 (Sam.688), 37 (Sam.744), 39 (Sam.753), 43 (Sam.787), 44 (Sam.805), 44 (Sam.796), 45 (Sam.809), 47 (Sam.825), 49

(Sam.844), 49 (Sam.834), 50 (Sam.837), 54 (Sam.878), 60 (Sam.911), 62 (Sam.919), 63 (Sam.924), 69 (Sam.966), 72 (Sam.978a), 73 (Sam.981), 76 (Sam.1004), 87 (Sam.1190)

+ Galium laconicum Boiss. \& Heldr. in Boiss. - H, BA - 72 (Sam.977)

+ Galium rotundifolium L. - H, EA - 17 obs. 
* Rubia peregrina L. - P, MA - B\&Q (1976:15)

+ Sherardia arvensis L. - T, EA - 15 (Sam.426), 125 (Sam.1146, Sam.1135), 126 (Sam.1103)

\section{SALICACEAE}

+ Populus alba L. - P, EA - obs.

+ Salix alba L. - P, EA - 130 (Sam.563)

+ Salix amplexicaulis Bory \& Chaub. in Bory - P, Me - 133 (Sam.536)

SANTALACEAE

+ Thesium divaricatum Mert. \& W. D. J. Koch - H, ME - 24 (Sam.625), 30 (Sam.679)

+ Viscum album subsp. abietis (Wiesb.) Abrom. in Wünsche - P, EA - obs.

\section{SAXIFRAGACEAE}

+ Saxifraga carpetana subsp. graeca (Boiss. \& Heldr.) D. A. Webb - H, Me - 2 (Sam.69), 28 (Sam.658, Sam.1263), 45 (Sam.815)

+ Saxifraga rotundifolia subsp. chrysosplenifolia (Boiss.) D. A. Webb - H, Bk - 119 (Sam.531)

\section{SCROPHULARIACEAE}

*Verbascum blattaria L. - H, EA - W\&W (2006:102)

+ Verbascum undulatum Lam. - H, Bk - 91 (Sam.1373)

SOLANACEAE

* Lycium chinense Mill. - P, X - W\&W (2006:77)

* Solanum nigrum L. - P, Co - W\&W (2006:94)

\section{URTICACEAE}

* Parietaria judaica L. \{= Parietaria diffusa Mert. \& W. D. J. Koch $\}$ - H, EA - W\&W (2008:122)

\section{VERBENACEAE}

*Verbena officinalis L. - H, Ct - W\&W (2006:103)

Vitex agnus-castus L. - P, MS - B\&Q (1976:15), W\&W (2006:104), obs.

\section{VERONICACEAE}

Digitalis ferruginea L. subsp. ferruginea - H, ME - B\&Q (1976:29), 13 (Sam.227), 72 (Sam.975), 76 (Sam.998, Sam.1087)

+ Digitalis grandiflora Mill. - H, Eu - 55 (Sam.882)

+ Digitalis laevigata subsp. graeca (Ivanina) Werner - H, Bk - 35 (Sam.730), 55 (Sam.883), 72 (Sam.976)

* Kickxia commutata subsp. graeca (Bory \& Chaub.) R. Fem. - H, EM - W\&W (2006:75)

* Linaria pelisseriana (L.) Mill. - T, MS - W\&W (2008:104)

+ Veronica anagallis-aquatica L. subsp. anagallis-aquatica - H T, Co - 134 (Sam.1212)

+ Veronica arvensis L. - T, EA - 1 (Sam.218), 32 (Sam.702), 34 (Sam.719), 38 (Sam.749), 56 (Sam.890), 89 (Sam.1222)

- Veronica chamaedrys subsp. chamaedryoides (Bory \& Chaub.) M.A. Fisch. in Strid \& Tan - H, Endem. - B\&Q (1976:29), 1 (Sam.219), 3 (Sam.220), Sam.221), 4 (Sam.222), 83 (Sam.1063)

+ Veronica cymbalaria Bodard - T, Me - 1 (Sam.223), 2 (Sam.224), 3 (Sam.225), 15 (Sam.430), 16 (Sam.459), 17 (Sam.475), 18 (Sam.492), 43 (Sam.785b), 79 (Sam.1255), 85 (Sam.1170)

+ Veronica hederifolia L. - T, EA - 3 (Sam.226), 78 (Sam.1243) 
VALERIANACEAE

+ Valerianella carinata Loisel. - T, EA - 15 (Sam.434), 79 (Sam.1253)

+ Valerianella coronata (L.) DC. - T, EA - 90 (Sam.1421), 97 (Sam.1112)

+ Valerianella locusta (L.) Laterr. - T, EA - 2 (Sam.301), 16 (Sam.462), 29 (Sam.1266), 85 (Sam.1176)

VIOLACEAE

+ Viola aetolica Boiss. \& Heldr. - H, Bk - 83 (Sam.1065)

+ Viola alba subsp. dehnhardtii (Ten.) W. Becker - H, Me - 3 (Sam.150, Sam.151), 4 (Sam.152), 10 (Sam.153), 11 (Sam.154), 46 (Sam.822)

+ Viola kitaibeliana Schult. in Roem. \& Schult. - T, ME - 90 (Sam.1399)

ZYGOPHYLACEAE

* Tribulus terrestris L. - T, Co - W\&W (2006:99)

SPERMATOPHYTA - ANGIOSPERMAE MONOCOTYLEDONEAE

ALLIACEAE

+ Allium guttatum subsp. tenorei (Parl.) Soldano $\{=$ A. guttatum subsp. sardoum (Moris) Stearn $\}$ - G, Me - 3 (Sam.358), 10 (Sam.359)

* Allium sphaerocephalon L. subsp. sphaerocephalon - G, ME - W\&W (2008:47)

AMARYLLIDACEAE

* Sternbergia lutea (L.) Spreng. subsp. lutea - G, MS - W\&W (2006:97)

ARACEAE

+ Arum cylindraceum Gasp. $\{=$ A. alpinum Schott \& Kotschy $\}$ - G, ME - 7 (Sam.302), 13 (Sam.303), 17 (Sam.480)

ASPARAGACEAE

Asparagus acutifolius L. - C, Me - B\&Q (1976:15), 5 (Sam.360), 85 (Sam.1155)

ASPHODELACEAE

+ Asphodelus ramosus L. subsp. ramosus \{“A. aestivus Brot.”\} - G, Me - 127 (Sam.552)

\section{CYPERACEAE}

+ Carex distachya Desf. - H, ME - 15 (Sam.441), 32 (Sam.699), 66 (Sam.952), 67 (Sam.959), 85 (Sam.1159)

+ Carex divulsa Stokes - H, EA - 86 (Sam.1177)

+ Carex flacca subsp. serrulata (Spreng.) Greuter in Greuter \& Rech. f. - G, MS - 3 (Sam.305, Sam.304), 17 (Sam.485), 85 (Sam.1152), 86 (Sam.1178), 89 (Sam.1225)

+ Carex pendula Huds. - H, EA - 135 (Sam.558)

\section{DIOSCOREACEAE}

+ Dioscorea communis (L.) Caddick \& Wilkin \{= Tamus communis L. $\}$ - G, ME - 17 (Sam.479), 35 (Sam.735), 82 (Sam.1050) 


\section{HYACINTHACEAE}

+ Muscari comosum (L.) Mill. - G, ME - 1 (Sam.361), 17 (Sam.481)

Prospero autumnale (L.) Speta - G, Me - W\&W (2006:91), 125 (Sam.1271)

IRIDACEAE

+ Iris tuberosa L. \{=Hermodactylus tuberosus (L.) Mill.\} - G, Me - 15 (Sam.439)

\section{JUNCACEAE}

Luzula forsteri (Sm.) DC. - H, Me - B\&Q (1976:29), 2 (Sam.357)

\section{ORCHIDACEAE}

+ Anacamptis morio subsp. caucasica (K. Koch) H. Kretzschmar, Eccarius \& H. Dietr. - G, MS obs.

+ Cephalanthera longifolia (L.) Fritsch - G, EA - 35 (Sam.736), 59 (Sam.910)

+ Cephalanthera rubra (L.) Rich. - G, EA - 14 (Sam.363), 76 (Sam.1007)

+ Dactylorhiza romana subsp. romana $\{=$ D. sambucina (L.) Soó subsp. pseudosambucina (Ten.) Sunderm.\} - G, Me - 26 (Sam.646), 28 (Sam.664, Sam.1260), 29 (Sam.672, Sam.1267), 39 (Sam.756), 49 (Sam.835)

+ Epipactis helleborine (L.) Grantz subsp. helleborine - G, Pt - 45 (Sam.818), 48 (Sam.830), 75 (Sam.992), 76 (Sam.1008), 83 (Sam.1066)

+ Epipactis microphylla (Ehrh.) Sw. - G, EA - 4 (Sam.365)

+ Limodorum abortivum (L.) Sw. - G, Me - 44 (Sam.803)

+ Neotinea maculata (Desf.) Stearn - G, Me - 33 (Sam.716), 54 (Sam.881), 57 (Sam.901), 66 (Sam.951), 16 (Sam.1092)

+ Neottia nidus-avis (L.) Rich. - G, EA - 41 (Sam.770), 44 (Sam.804), 75 (Sam.994)

* Ophrys apifera Huds. - G, ME - H (1985)

* Ophrys helenae Renz - G, Bk - T\&A (2017)

* Ophrys holoserica (Burm. f.) Greuter subsp. holoserica - G, Me - H (1985) Ophrys sphegodes subsp. mammosa (Desf.) Soo ex E. Nelson - G, ME - obs., K (1983), H (1985), T\&A (2017)

Ophrys sphegodes subsp. spruneri (Nyman) E. Nelson - G, EM - obs., H (1985), T\&A (2017)

* Ophrys tenthredinifera Willd. - G, Me - T\&A (2017)

+ Ophrys umbilicata Desf. subsp. umbilicata - G, Me - obs.

* Orchis anthropophora (L.) All. - G, MA - T\&A (2017) Orchis italica Poir. in Lam. \& al. - G, Me - 103 (Sam.1236), T\&A (2017)

+ Orchis provincialis Lam. \& DC. - G, ME - 28 (Sam.665), 29 (Sam.1268), 39 (Sam.758), 43 (Sam.784), 44 (Sam.801), 48 (Sam.831), 54 (Sam.880), 55 (Sam.887), 57 (Sam.900), 58 (Sam.903), 59 (Sam.909), 74 (Sam.989), 81 (Sam.1047)

+ Platanthera chlorantha (Custer) Rchb. in Mössler - G, ES. - 28 (Sam.663), 35 (Sam.737) Spiranthes spiralis (L.) Chevall. - G, EA - 85 (Sam.1977), T\&A (2017)

\section{POACEAE}

Achnatherum bromoides (L.) P. Beauv. \{= Stipa bromoides (L.) Dörfl. $\}$ - H, Me - B\&Q (1976:15), 3 (Sam.354, Sam.355), 15 (Sam.442), 16 (Sam.466), 19 (Sam.515), 22 (Sam.602), 66 (Sam.953), 89 (Sam.1229)

+ Aegilops biuncialis Vis. subsp. biuncialis $\{=$ A. lorentii Hochst. $\}-$ T, MS - 90 (Sam.1408)

+ Aegilops geniculata Roth - T, Me - 125 (Sam. 1145)

+ Aira elegantissima Schur - T, MS - 30 (Sam.678), 31 (Sam.690)

+ Anthoxanthum odoratum L. - H, Co - 5 (Sam.306), 15 (Sam.446) 
Arrhenatherum elatius (L.) J. Presl \& C. Presl - H, Pt - B\&Q (1976:29), 2 (Sam.307), 3 (Sam.308, Sam.309,), 4 (Sam.310), 12 (Sam.314)

Brachypodium pinnatum (L.) P. Beauv. - H, ES - B\&Q (1976:29), 3 (Sam.318), 40 (Sam.761) Brachypodium sylvaticum (Huds.) P. Beauv. subsp. sylvaticum - H, Pt - B\&Q (1976:29), W\&W (2008:59), 4 (Sam.326), 15 (Sam.443), 17 (Sam.483)

+ Briza maxima L. - T, ST - 25 (Sam.637)

* Bromus sterilis L. - T, MS - W\&W (2008:61)

+ Bromus tectorum L. - T, Pt - 90 (Sam.1414)

* Catapodium rigidum (L.) C. E. Hubb. - T, Me - W\&W (2008:67)

Cynosurus echinatus L. - T, Me - W\&W (2008:79), 3 (Sam.330), 5 (Sam.331)

Cynosurus effusus Link. \{= C. elegans Desf. $\}$ - T, Me - B\&Q (1976:29), 4 (Sam.329), 12 (Sam.332), 15 (Sam.445), 16 (Sam.1238), 21 (Sam.528), 28 (Sam.1262), 88 (Sam.1195)

Dactylis glomerata subsp. hispanica (Roth) Nyman - H, Me - B\&Q (1976:29), 1 (Sam.334), 3 (Sam.335, Sam.336), 4 (Sam.337)

+ Dasypyrum villosum (L.) P. Candargy - T, MS - 90 (Sam.1410)

* Digitaria sanguinalis (L.) Scop. subsp. sanguinalis - T, Co - W\&W (2006:63)

+ Elymus caninus (L.) L. \{=Agropyron caninum L. - H, ES - 22 (Sam.602), 23 (Sam.607)

+ Elymus panormitanus (Parl.) Tzvelev $\{=$ Roegneria panormitana (Parl.) Nevski $\}$ - H, Me - 3 (Sam.338), 46 (Sam.821)

* Eragrostis cilianensis (All.) Janch. subsp. cilianensis - T, Co - W\&W (2006:68)

* Eragrostis minor Host subsp. minor - T, Co - W\&W (2006:68)

* Eragrostis pilosa (L.) P. Beauv. - T, Co - W\&W (2006:68)

+ Festuca heterophylla Lam. - H, Eu - 6 (Sam.340), 35 (Sam.738), 37 (Sam.745), 39 (Sam.759), 44 (Sam.806), 47 (Sam.826), 49 (Sam.836, Sam.846), 55 (Sam.888), 57 (Sam.902), 60 (Sam.915), 63 (Sam.925), 68 (Sam.961), 71 (Sam.973), 72 (Sam.980), 129 (Sam.781)

+ Festuca valesiaca subsp. valesiaca - H, EA - 14 (Sam.342), 75 (Sam.995), 78 (Sam.1028)

* Holcus lanatus L. - H, ES - W\&W (2008:95)

* Hordeum murinum L. subsp. murinum - T, MS - W\&W (2008:96)

+ Koeleria lobata (M. Bieb.) Roem. \& Schult. $\{=$ K. splendes C. Presl $\}-\mathrm{H}, \mathrm{Me}-90$ (Sam.1412)

* Lolium rigidum Gaudin subsp. rigidum - T, ST - W\&W (2008:106) Melica uniflora Retz. - H, Pt - B\&Q (1976:29), 11 (Sam.344), 14 (Sam.346)

+ Phleum phleoides (L.) H. Karst. $\{=$ P. montanum K. Koch $\}$ - H, Eurosib. - 42 (Sam.777), 54 (Sam.879), 90 (Sam.1409)

* Phleum subulatum (Savi) Asch. \& Graebn. - T, Me - W\&W (2008:124)

* Poa annua L. - T, Co - W\&W (2008:127)

+ Poa bulbosa L. subsp. bulbosa - H, Pt - 2 (Sam.347, 12 (Sam.348), 15 (Sam.440), 16 (Sam.465), 19 (Sam.514), 28 (Sam.1265), 38 (Sam.750), 85 (Sam.1175), 89(Sam.1227)

* Poa nemoralis L. subsp. nemoralis - H, ES - B\&Q (1976:29)

+ Poa trivialis subsp. sylvicola (Guss.) H. Lindb. - H, EA - 1 (Sam.349), 2 (Sam.350), 3 (Sam.351, Sam.352), 17 (Sam.482), 18 (Sam.498), 22 (Sam.603), 24 (Sam.626), 38 (Sam.751), 46 (Sam.820), 81 (Sam.1046), 85 (Sam.1167), 89 (Sam.1218)

+ Secale strictum (C. Presl) C. Presl subsp. strictum $\{=$ S. montanum Guss. $\}$ - H, MS - 90 (Sam.1413)

* Setaria viridis (L.) P. Beauv. subsp. viridis - T, Co - W\&W (2006:92)

* Sorghum halepense (L.) Pers. - G, X - W\&W (2006:96)

+ Taeniatherum caput-medusae subsp. crinitum (Schreb.) Melderis - T, Me - 90 (Sam.1406)

* Tragus racemosus (L.) All. - T, Co - W\&W (2006:99)

\section{RUSCACEAE}

Ruscus aculeatus L. - G C, ME - B\&Q (1976:15), 2 (Sam.362) 
Table 1. Numbers of plant families, genera, species, subspecies and taxa in the three main taxonomic groups of the vascular flora of Mt. Goulinas.

\begin{tabular}{lccccc}
\hline & Families & Genera & Species & Subspecies & $\begin{array}{c}\text { Specific and } \\
\text { subspecific } \\
\text { taxa }\end{array}$ \\
\hline Pteridophytes & 4 & 4 & 6 & 2 & 6 \\
Gymnosperms & 3 & 3 & 3 & 1 & 3 \\
Angiosperms & 74 & 278 & 475 & 139 & 478 \\
Total & 81 & 285 & 484 & 142 & 487 \\
\hline
\end{tabular}

Spectra and notes on the floristic catalogue - The so far known vascular flora of Mt Goulinas consists of 487 taxa, belonging to 81 families and 285 genera (Table 1). From these, 381 taxa were either collected or observed by the first author, while 106 literature references could not be confirmed. From the total number of taxa, 305 are reported for the first time. Among the new references we can mention Euphorbia phymatosperma subsp. cernua and Elymus caninus, two taxa that are reported for the first time from Sterea Ellas. Campanula sparsa subsp. sphaerothrix, although not confirmed for Mt Goulinas, was collected by the first author from the neighbour Mt Oxia (unpublished material) and therefore the report from Barbéro \& Quézel (1976) may be correct. Six (6) Greek endemics are reported from Mt Goulinas, five (5) of them for the first time: Abies cephalonica, Hieracium bracteolatum subsp. reinholdii, Alkanna calliensis, Erysimum asperulum and

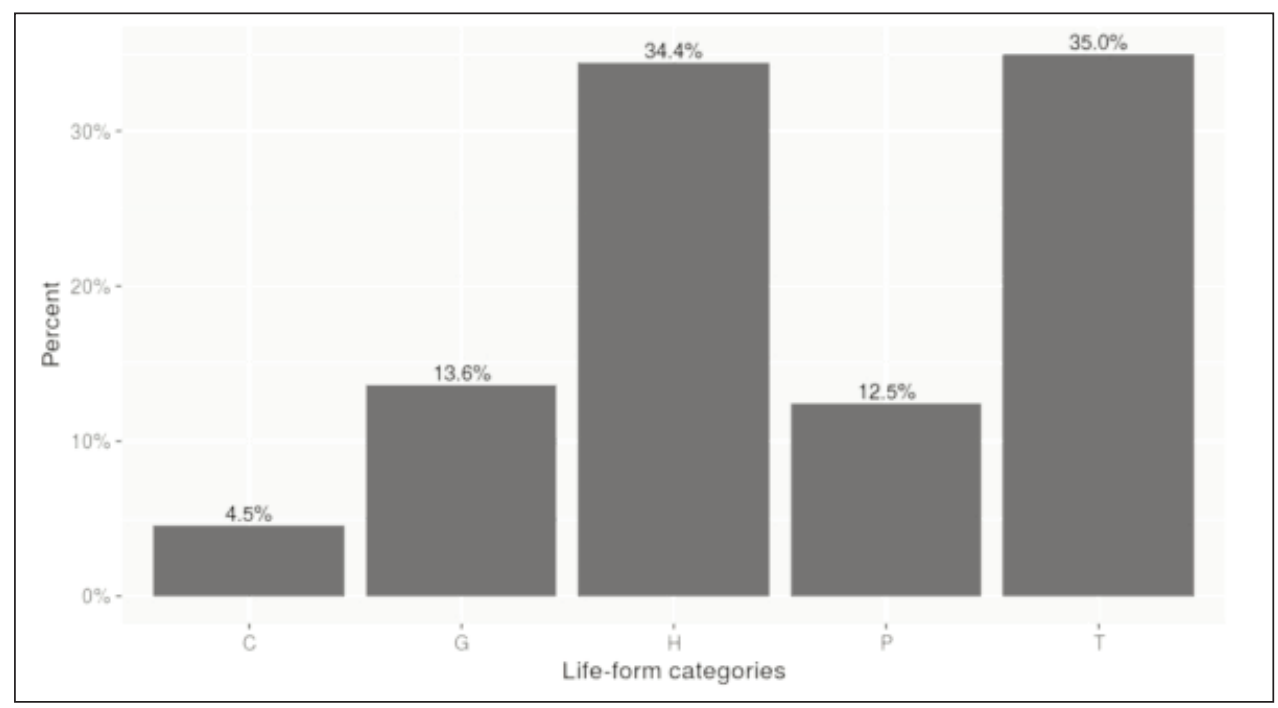

Fig. 2. Life-form spectrum of the vascular flora of Mt Goulinas. P: Phanerophytes, C: Chamaephytes, H: Hemicryptophytes, G: Geophytes, T: Therophytes. 


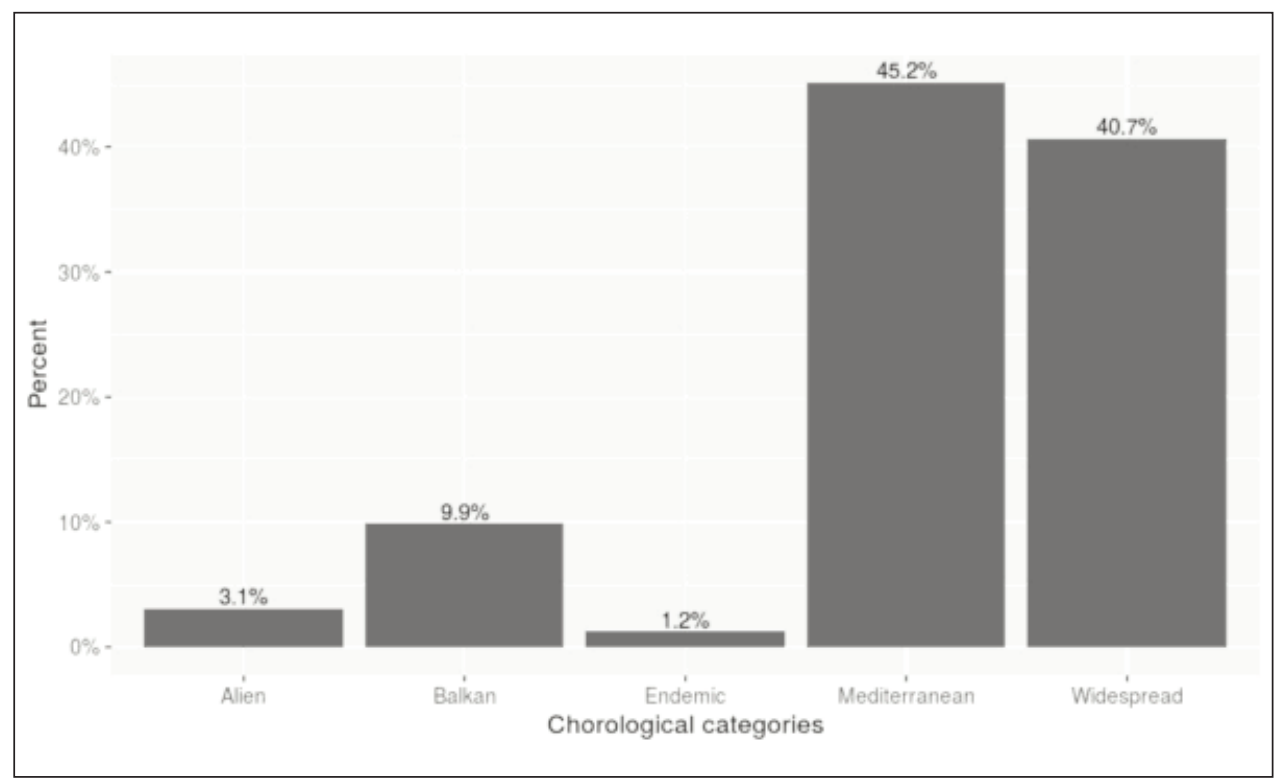

Fig. 3. Chorological spectrum of the vascular flora of Mt Goulinas.

Stachys graeca. Forty eight (48) Balkan and sub-Balkan endemics are also reported, 36 of which are new records for the study area.

Hemicryptophytes and therophytes are the most abundant plant life forms in the flora of Mt Goulinas with a proportion of $34.4 \%$ and $35 \%$ respectively (Fig. 2). Geophytes and phanerophytes follow with $13.6 \%$ and $12.5 \%$ respectively, while chamaephytes are by far the less abundant $(4.5 \%)$. The most abundant chorological group of plants are the Mediterranean taxa (220 taxa $-45.2 \%)$, followed by the Widespread taxa (198 taxa -40.7 \%) (Fig. 3). The proportion of Balkan taxa, Greek endemics and Aliens is much lower (9.9 $\%, 1.2 \%$ and $3.1 \%$ respectively).

\section{Acknowledgements}

We are grateful to Günter Gottschlich for verifying or identifying the specimens of the genus Hieracium.

\section{References}

Barbéro, M. \& Quézel, P. 1976: Les groupements forestiers de Grèce Centro-Méridionale. - Ecol. Medit. 2: 3-86.

Baumann, H., Künkele, S. \& Lorenz, R. 2006: Orchideen Europas mit angrenzenden gebieten. Stuttgart.

Boyce, P. 1988: A new classification of Arum with keys to the infrageneric taxa. - Kew Bull. 44: 383-395. doi: 10.2307/4110359 
- 1994: The genus Arum (Araceae) in Greece and Cyprus. - Ann. Mus. Goulandris 9: 27-38.

Christensen, K. I. 1992: Revision of Crataegus Sect. Crataegus and Nothosect. Crataeguineae (Rosaceae-Maloideae) in the Old World. - Syst. Bot. Monogr. 35: 1-199. doi: $10.2307 / 25027810$

Dimopoulos, P., Raus, Th., Bergmeier, E., Konstantinidis, T., Iatrou, G., Kokkini, S., Strid, A. \& Tzanoudakis, D. 2013: Vascular plants of Greece: An annotated checklist. - Berlin \& Athens.

Hölzinger, J., Künkele, A., Künkele, S. 1985: Die Verbreitung der Gattung Ophrys L. auf dem griechischen Festland. - Mitteilungsbl. Arbeitskr. Heim. Orch. Baden-Wurttemberg 17: 1-101.

Kallergis, G. A., Koch, K. E. \& Nikolaus, H. J. 1970: Geological map of Greece. - Athens

Krendl, F. 1986: Die Arten Der Galium mollugo-Gruppe in Griechenland. - Bot. Chron. 6-7: 5-170.

Künkele, S. 1983: Zum Stand der Orchideenkartierung in Griechenland. - Mitteilungsbl. Arbeitskr. Heim. Orch. Baden-Wurttemberg 15: 11-42.

Phitos, D. 1960: Phytogeographic observations in the Timfristos - Oxia mountain range [in Greek]. - Dasika Chron. 25: 1099-1105.

Samaras, D., Theodoropoulos, K. \& Eleftheriadou, E. 2008: The plant communities of Greek fir forests (Abies cephalonica J.W. Loudon) of Mt. Goulinas (C. Greece). [in Greek with English abstract]. - Forest Res. 21: 63-78.

Strid, A. (ed.) 1986: Mountain Flora of Greece, 1. - Cambridge.

— \& Tan, K. (eds.) 1991: Mountain Flora of Greece, 2. - Edinburgh.

— \& Tan, K. (eds.) 1997: Flora Hellenica, 1. - Königstein.

— \& Tan, K. (eds.) 2002: Flora Hellenica, 2. - Ruggell.

Tsiftsis, S., Antonopoulos, Z. 2017: Atlas of the Greek Orchids, 2. - Rethymno.

Tutin, T. G., Burges, N. A., Chater, A. O., Edmondson, J. R., Heywood, W. H., Moore, D. M., Valentine, D. H., Walters, S. M. \& Webb, D. A. (eds.) 1993: Flora Europaea, 1. - Cambridge.

- , Heywood, V. H., Burges, N. A., Moore, D. M., Valentine, D. H., Walters, S. M. \& Webb, D.A. (eds.) 1968-1980: Flora Europaea, 2-5. - Cambridge.

Willing, A. \& Willing, E. 2006: A Willing contribution to Flora Hellenica. Field records autumn 2005. - Berlin.

— 2008: A Willing contribution to Flora Hellenica. Field records 2007. - Berlin.

Zielinski, J. 1990: The genus Rosa L. in Greece. - Arbr. Kornickie 35: 3-45.

Zohary, M. \& Heller, D. 1984: The genus Trifolium. - Israel.

Addresses of the authors:

Dimitrios A. Samaras ${ }^{1,2}$, Eleni Eleftheriadou ${ }^{1}$, Konstantinos Theodoropoulos ${ }^{1}$ \& Georgios Karetsos ${ }^{2}$,

${ }^{1}$ Aristotle University of Thessaloniki, School of Forestry and Natural Environment, Laboratory of Forest Botany - Geobotany, P.O. Box 270, University Campus, GR54124, Thessaloniki, Greece

${ }^{2}$ Hellenic Agricultural Organization «DEMETER», Institute of Mediterranean Forest Ecosystems, P.O. Box 14180, Terma Alkmanos, Ilisia, GR-11528, Athens, Greece Corresponding author: Dimitrios A. Samaras, E-mail: mail@dimitrios-samaras.net 